\title{
A mechanical verification of the independence of Tarski's Euclidean axiom
}

\author{
by \\ Timothy James McKenzie Makarios
}

A thesis

submitted to the Victoria University of Wellington in fulfilment of the requirements for the degree of

Master of Science in Mathematics.

Victoria University of Wellington 2012 


\begin{abstract}
This thesis describes the mechanization of Tarski's axioms of plane geometry in the proof verification program Isabelle. The real Cartesian plane is mechanically verified to be a model of Tarski's axioms, thus verifying the consistency of the axiom system.

The Klein-Beltrami model of the hyperbolic plane is also defined in Isabelle; in order to achieve this, the projective plane is defined and several theorems about it are proven. The Klein-Beltrami model is then shown in Isabelle to be a model of all of Tarski's axioms except his Euclidean axiom, thus mechanically verifying the independence of the Euclidean axiom - the primary goal of this project.

For some of Tarski's axioms, only an insufficient or an inconvenient published proof was found for the theorem that states that the KleinBeltrami model satisfies the axiom; in these cases, alternative proofs were devised and mechanically verified. These proofs are described in this thesis - most notably, the proof that the model satisfies the axiom of segment construction, and the proof that it satisfies the five-segments axiom. The proof that the model satisfies the upper 2-dimensional axiom also uses some of the lemmas that were used to prove that the model satisfies the five-segments axiom.
\end{abstract}




\section{Acknowledgements}

Many people have assisted me while I was researching and writing this thesis; I wish to thank a few of them here.

First, I thank my supervisor, Professor Rob Goldblatt, for the publications he lent me or recommended to me, his advice about what to concentrate on, his advice on drafts of my thesis, and his general encouragement.

During my research, Dr Ken Pledger recommended a number of useful publications he was aware of; he is also the person whose teaching initially inspired my interest in geometry, for which I am very grateful.

Victoria University's Scholarships Office also deserves thanks. Because of my health, I was able to study only part-time, which disqualified me from most scholarships according to their ordinary rules; the Scholarships Office found a solution that eased my financial situation during my study.

More personally, I thank my family (both natural and -in-law) for their practical and moral support, which included some proof-reading. I particularly thank my wife, Deborah, who has loved and supported me in more ways than I can possibly mention here.

Finally, I thank God, who has made me everything that I am. 


\section{Contents}

1 Introduction 1

1.1 Euclid's Elements and the axiomatic method . . . . . . . . 1

1.2 Consistency and independence ... . . . . . . . . 3

1.3 Independence of the parallels postulate . . . . . . . . . 3

1.4 Mechanical proof verification ............ 5

1.5 Thesis outline ................. . . 7

1.6 Previous related work ............. 8

2 The choice of an axiom system 9

2.1 Euclid's axioms . . . . . . . . . . . . . . . 9

2.2 Hilbert's Grundlagen . . . . . . . . . . . . . . . . 11

2.3 Tarski's axioms . . . . . . . . . . . . . . . . . . 12

2.4 Statement and explanation of Tarski's axioms . . . . . . 13

3 The choice of a prover $\quad 20$

3.1 Wiedijk's Seventeen Provers . . . . . . . . . . . . . . . . . 20

3.2 Essential features . . . . . . . . . . . . . . . . 21

3.3 Desirable features . . . . . . . . . . . . . . . 22

3.4 Features useful for this project . . . . . . . . . . . . 24

3.5 Isabelle . . . . . . . . . . . . . . . . . . 25

4 Locales $\quad 27$

4.1 Overview ....................... 27 
4.2 A locale for Tarski's first three axioms . . . . . . . . . . 28

4.3 A locale for Tarski's first five axioms . . . . . . . . . . 28

4.4 A simple proof in a locale . . . . . . . . . . . . . . 29

4.5 The benefit of many locales . . . . . . . . . . . 30

4.6 Locales for the remaining axioms . . . . . . . . . . . 31

5 The consistency of Tarski's axioms 33

5.1 The model . . . . . . . . . . . . . . . . . 33

5.2 Congruence in semimetric spaces . . . . . . . . . . . . . . . . . . . . . . 35

5.3 The five-segments axiom . . . . . . . . . . . . . 36

5.4 The axiom of continuity . . . . . . . . . . . . 37

5.5 The Euclidean axiom . . . . . . . . . . . . . . 40

6 The independence of the Euclidean axiom 42

6.1 Existing proofs . . . . . . . . . . . . . . . . . . 42

6.2 Choosing the model . . . . . . . . . . . . . 44

$\begin{array}{lll}7 & \text { Formalizing the projective plane } & 47\end{array}$

7.1 Overview ...................... . . 47

7.2 Points of the projective plane . . . . . . . . . . . . . . 48

7.3 Abstraction and representation ... . . . . . . . . . . . . . . . . . . . . . . . . 59

7.4 Lines in the projective plane . . . . . . . . . . . . . 52

7.5 Collineations of the projective plane . . . . . . . . . . 54

7.6 A mapping to the Cartesian plane . . . . . . . . . . 56

8 Formalizing the hyperbolic plane $\quad 58$

8.1 Defining the model . . . . . . . . . . . . . 58

8.1.1 Preliminary results . . . . . . . . . . . 59

8.2 The reflexivity axiom for equidistance . . . . . . . . . . 60

8.2.1 Abstraction and representation in the proof of Statement $66 \ldots \ldots \ldots$.................. 61

8.2.2 Proof that Statement 66 and axiom 1 hold . . . . . 63 
8.3 Some betweenness-only axioms . . . . . . . . . . . . . 64

8.4 The axiom of segment construction . . . . . . . . . 66

8.4 .1 Statement $62 \ldots \ldots \ldots 66$

8.4 .2 Statement $63 \ldots \ldots \ldots \ldots 68 \ldots \ldots \ldots$

8.4.3 Proof that axiom 4 holds ........... 70

8.5 The five-segments axiom . . . . . . . . . . . . . 71

8.5 .1 Perpendicularity ............... 72

8.5 .2 Cross ratios ................. 72

8.5 .3 Distance .................... 73

8.5.4 A formula for a cross ratio involving a perpendicular foot . . . . . . . . . . . . . 75

8.5.5 Proof that Statement 69 and axiom 5 hold . . . . . 76

8.6 The upper 2-dimensional axiom . . . . . . . . . 78

8.6.1 Non-categoricity of Borsuk and Szmielew's axioms . 78

8.6.2 Proof that axiom 9 holds . . . . . . . . . . . 79

8.7 The negation of the Euclidean axiom . . . . . . . . . . 81

9 Conclusion $\quad 83$

9.1 Success ......................... 83

9.2 Equivalent axioms . . . . . . . . . . . . . . 84

9.3 Other possible future work . . . . . . . . . . . . . 84 


\section{List of Figures}

1.1 An illustration of Euclid's fifth postulate . . . . . . . . 2

1.2 An illustration of Playfair's axiom . . . . . . . . . . 4

2.1 Tarski's axiom 4 - the axiom of segment construction . . . 14

2.2 Tarski's axiom 5 - the five-segments axiom . . . . . . . 15

2.3 Tarski's axiom 7 - the axiom of Pasch . . . . . . . . 15

2.4 Tarski's axiom 9 - the upper 2-dimensional axiom . . . . 17

2.5 Tarski's axiom 10 - the Euclidean axiom . . . . . . . . . 17

2.6 Tarski's axiom 11 the axiom of continuity . . . . . . . 18

4.1 Tarski's first three axioms formalized in Isabelle . . . . . . . 28

4.2 A locale for Tarski's first five axioms . . . . . . . . . . . . . 29

4.3 A short proof in Isabelle . . . . . . . . . . . . . . . . . 29

4.4 A locale for Tarski's axioms of absolute geometry . . . . . . 31

4.5 A locale for Tarski's axioms of absolute plane geometry . . 32

4.6 A locale for Tarski's axioms of Euclidean space . . . . . . . . 32

4.7 A locale for Tarski's axioms of the Euclidean plane . . . . . 32

5.1 Definitions of congruence and betweenness in $\mathbb{R}^{n} \ldots$. . . 34

5.2 Semimetric spaces satisfy Tarski's first three axioms . . . . . 35

5.3 Beginning to verify the continuity axiom . . . . . . . . 37

5.4 Degenerate cases considered simultaneously . . . . . . . . 39

5.5 Measuring distances from $a$ using the segment $a c \ldots 39$

5.6 A construction for Tarski's Euclidean axiom . . . . . . . . . 41 
7.1 Definition of proportionality and non-zero vectors . . . . . . 48

7.2 Defining a type for the points of the real projective plane . . 48

7.3 Useful abstraction and representation functions for proj2 . . 50

7.4 Abstracting a representative . . . . . . . . . . 51

7.5 Defining a type for lines of the projective plane . . . . . 53

7.6 Abstraction and representation functions for proj2-line . . . 53

7.7 Abstracting a representative using a similar theorem . . . . 54

7.8 Applying a collineation to a point . . . . . . . . . . . 55

7.9 Applying a collineation to a line . . . . . . . . . . . . . 55

7.10 A map from the projective plane to the Cartesian plane . . . 56

7.11 proj2-pt and cart2-pt are inverses . . . . . . . . . . 57

8.1 Lifting betweenness from the Cartesian plane . . . . . . . . 59

8.2 Constructions for the proof of Statement 66 . . . . . . . . 62

8.3 Constructions for the verification of axiom $1 \ldots 64$

8.4 Lifting axiom 6 from the Cartesian plane . . . . . . . . . 65

8.5 Constructions for the verification of axiom $4 \ldots 70$

8.6 Constructions for the proof of Statement 69 . . . . . . . . 76

8.7 Constructions for the verification of axiom 9 . . . . . . 80

8.8 The model satisfies the axioms of absolute plane geometry . 81

8.9 A counter-example to the Euclidean axiom . . . . . . . . . 82

8.10 The model does not satisfy all of the axioms . . . . . . . 82 


\section{Chapter 1}

\section{Introduction}

\subsection{Euclid's Elements and the axiomatic method}

Approximately twenty-three centuries ago, Euclid wrote his Elements, possibly history's most enduring textbook in mathematics - or, indeed, in any subject - (see [7, pages v-vi, 2]). The Elements documents an impressive breadth of ancient mathematics, starting from geometry and framing other topics in geometrical ways (see [23, pages 103-104]). One important feature of Euclid's work was his pioneering use of the axiomatic method.

The axiomatic method, understood in modern terms, requires that the mathematician states from the outset exactly what they will assume (the axioms); then they may derive logical consequences from the axioms, but they may not make any other assumptions, either implicitly or explicitly. This ensures that if the axioms are true of some mathematical structure (called a model), then all the consequences must also be true of the model.

Euclid's axioms are divided into two groups, which T. L. Heath's translation calls postulates and common notions (see [7, pages 154-155]). The common notions are primarily concerned with the nature of equality, but the postulates are more geometrical in nature. Quoting from [7], the postulates are: 


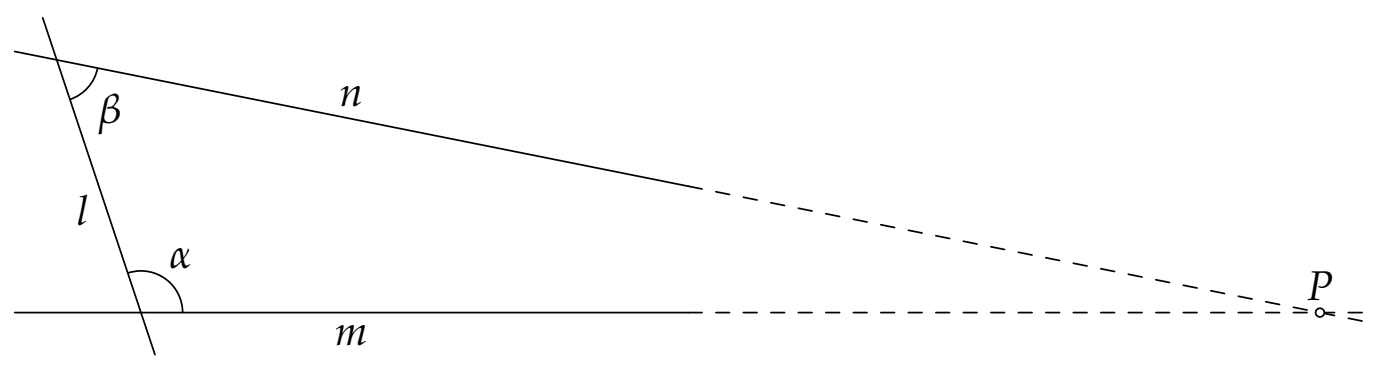

Figure 1.1: An illustration of Euclid's fifth postulate

1. To draw a straight line from any point to any point.

2. To produce a finite straight line continuously in a straight line.

3. To describe a circle with any centre and distance.

4. That all right angles are equal to one another.

5. That, if a straight line [ $l]$ falling on two straight lines [ $m$ and $n$ ] make the interior angles [ $\alpha$ and $\beta$ ] on the same side [of $l]$ less than two right angles [when added together], the two straight lines, if produced indefinitely, meet [at $P$ ] on that side [of $l$ ] on which are the angles less than the two right angles.

To a modern mathematician, the first three postulates look somewhat strange as axioms, since they appear to postulate a procedure, rather than a proposition; such a mathematician might be more inclined to assert the existence of a straight line from any point to any point in the first postulate, for example.

To aid in understanding the fifth postulate, I have constructed Figure 1.1 and inserted matching labels and other explanatory phrases into the statement of the postulate. The fifth postulate is often called the "parallels postulate", since it prescribes a situation in which two lines ( $m$ and $n$ in Figure 1.1) must not be parallel (and has many consequences regarding lines that are parallel). This postulate should be understood as referring to the behaviour of lines in a plane, not lines in higher dimensions. 


\subsection{Consistency and independence}

Two important properties related to axiom systems are consistency and independence.

A set of axioms is said to be consistent if no logical contradictions can be derived from the axioms. One way of establishing the consistency of an axiom system is to exhibit a model of the axioms. Then any contradiction that can be derived from the axioms must imply a contradiction in the logical setting in which the model was defined. Consistency is important because without it, everything is provable from the axioms, and we learn nothing from any of the proofs.

Within an axiom system, a particular axiom, (A), is said to be independent of the other axioms if it is not a logical consequence of the other axioms. The independence of (A) can be established by exhibiting a model of the other axioms that does not satisfy (A); if (A) can be false when all the other axioms are true, then it cannot possibly be a logical consequence of the other axioms.

Independence is of interest because if an axiom is not independent, then it can be removed from the axiom system without reducing that system's power; the truth of the removed axiom can be proven from the other axioms, allowing the subsequent proof of other theorems whose proofs might have used that axiom. The simplified axiom system may then better elucidate the essence of the theory it defines, and fewer axioms must be checked when establishing that a particular model satisfies the axioms.

\subsection{Independence of the parallels postulate}

For many centuries, various mathematicians were suspicious of the parallels postulate, believing that it was in the nature of a theorem requiring proof, rather than an axiom. (Axioms were, for much of the history of 
$=\frac{P}{?}$

l

Figure 1.2: An illustration of Playfair's axiom

mathematics, regarded as self-evident truths, rather than somewhat arbitrary propositions used as the basis of an abstract theory, ideally consistent, independent, and interesting.)

Numerous mathematicians believed that they had proven the parallels postulate from Euclid's other axioms, thus proving that it was not independent. Such "proofs" range from Claudius Ptolemy's "proof" in the second century to Adrien-Marie Legendre's "proofs" in the eighteenth and nineteenth centuries (see [7, pages 204-219] and [8, page 304]). All such attempted proofs involved an extra assumption (usually equivalent to the parallels postulate), but the mathematicians in question seldom realized that they were making an unjustified assumption.

Honourable mention should go to Omar Khayyám, the first mathematician to deliberately and explicitly assume an alternative axiom and prove that the parallels postulate was a consequence of that axiom - in the context of Euclid's other axioms (see [20, page 64]).

Perhaps the most famous alternative axiom equivalent to the parallels postulate is the one known as Playfair's axiom (although John Playfair himself wrote of earlier use - see [8, page 303]). Playfair stated it like this:

That two straight lines, which cut one another, cannot be both parallel to the same straight line.

Equivalently, given a line $l$ and a point $P$ not on $l$, there is at most one line 
through $P$ that does not intersect $l$. This is illustrated in Figure 1.2, where the axiom asserts that either $m$ or $n$ (or perhaps both) must intersect $l$, when sufficiently extended.

In the nineteenth century, a number of mathematicians - most notably János Bolyai and Nikolai Ivanovich Lobachevsky, who worked independently - believed (in modern terms) that the parallels postulate was independent of Euclid's other axioms (see [3, page 4]). They assumed the negation of the parallels postulate and systematically investigated the consequences. However, their work was not mainstream; according to [3, page 4], "they were regarded as eccentric and pathological".

Finally, in 1868, Eugenio Beltrami provided several models of what is now known as hyperbolic geometry (see, for example [2]). These models satisfy all of Euclid's axioms except the parallels postulate, thus establishing that the parallels postulate is independent. Within hyperbolic geometry, Beltrami also provided a model of all of Euclid's axioms, establishing that Euclidean geometry (where the parallels postulate holds) is just as consistent as hyperbolic geometry; a logical contradiction in one would imply a logical contradiction in the other.*

\subsection{Mechanical proof verification}

Although the axiomatic method adds rigour to mathematics, it relies on human mathematicians to apply it flawlessly. As can be seen by the number of flawed "proofs" of the non-independence of the parallels postulate (see Section 1.3), human mathematicians are all too likely to make unjustified assumptions. Their readers can easily fall into the same reasoning flaws; Playfair, for example, believed at least one of Legendre's "proofs"

\footnotetext{
*Beltrami probably did not understand consistency and independence in the modern sense; for a discussion of the emergence of these ideas, see [25]. Nevertheless, Beltrami was the first to provide these models, from which a modern mathematician may immediately deduce the independence of the parallels postulate.
} 
of the non-independence of the parallels postulate (see [8, page 305]).

To mitigate human fallibility, computers are now sometimes used for proof verification, taking the rigour of the axiomatic method even further. Provided that a computer is programmed correctly and does not suffer from physical faults, it can infallibly strictly apply the axioms and allowable rules of inference that it is given. A mathematician who attempts to provide a faulty proof to such a proof verification program will inevitably fail, and, with luck, will realize their error sooner, rather than later.

Because computers and their programmers can also be fallible, mechanical proof verification does not give complete certainty. However, it can provide much more certainty than merely human-checked proofs.

A number of techniques can be used to increase the certainty given by mechanical verification. For example, the program could be run several times on different computers, to mitigate the possibility of hardware faults. It is also possible to write independent proof verification programs that read the same proof scripts, lessening the likelihood that an unnoticed error in the program will permit a mathematician to make an unnoticed logical error. So-called "soft errors" caused by cosmic rays (see [30]) could be mitigated by making proofs (and their checking process) modular. This would ensure that individual parts of a proof are checked quickly; if they are checked twice, it would be vanishingly unlikely that a soft error occurred during both checks.

Proof verification should be distinguished from other mathematical uses of computers. Specifically, it differs from computation and proof search. Although both computation and proof search can be used in proof verification systems, their intent is different.

It is particularly important to distinguish between proof search and proof verification. Proof search involves the computer finding a proof; proof verification requires a human mathematician (or, indeed a proof search program) to provide in meticulous detail the proof that is to be verified. If a proof search program finds a valid proof of a desired theo- 
rem, it has served its purpose, even if the program has errors; an error in a proof verification program, however, may put into doubt the validity of every judgement the program has ever made.

\subsection{Thesis outline}

There is a somewhat arbitrary list of 100 famous mathematical theorems at [34]. This list indicates which of those theorems have been mechanically verified. At the time of writing this thesis, the list shows that thirteen of the theorems have not yet been mechanically verified. One of the unverified theorems is the independence of the parallels postulate. This thesis describes what I believe to be the first mechanical verification of this theorem.

This thesis begins by describing the choice of an axiom system for geometry (in Chapter 2), and the choice of a proof verification program (in Chapter 3). Then, Chapter 4 explains how the axioms were formalized in the proof verification program.

Chapter 5 describes how a model of Euclidean geometry is defined and used to mechanically verify the consistency of the chosen system of axioms (relative to the correctness of the proof verification program and the consistency of the logic it implements).

Chapter 6 explains the choice of a model of the hyperbolic plane; this second model requires the formalization of the projective plane, described in Chapter 7, and, as explained in Chapter 8, it is used to mechanically verify the independence of the axiom that is equivalent to the parallels postulate.

Finally, Chapter 9 summarizes what has been achieved, and notes possible future work in this area. 


\subsection{Previous related work}

Some impressive work has already been done in mechanizing geometry. For example, Shang-Ching Chou, Xiao-Shan Gao, and Jing-Zhong Zhang have published work on mechanical proof search in Euclidean geometry (see, for example, [4]). This differs from the goals of my work in a number of ways: for example, it is work in proof search, as opposed to proof verification; also, it is concerned with the consequences of the axioms, rather than models of the axioms.

Similarly, much of the existing work in proof verification in geometry is also concerned with the consequences of sets of geometrical axioms; see, for example, [15], [16], and [22]. In particular, [15, page 333] explicitly notes that future work might involve the construction of a model of their chosen set of axioms for geometry.

I am aware of one example of a mechanical verification that a particular model of geometry satisfies a certain set of axioms. Jacques Fleuriot chose some axioms based on those of Chou and his collaborators (see [9, pages 22-23]); then, in a proof verification program called Isabelle ${ }^{\dagger}$, he defined a model of the axioms and proved that it was indeed a model (see [9, pages 67-74]). It is worth noting that his chosen axioms are not categorical; that is, there are multiple inequivalent (technically, non-isomorphic) models of the axioms. Indeed, Fleuriot's model is not the standard real Cartesian model of Euclidean geometry; he uses the hyperreals, in order to write mechanically verifiable proofs that more faithfully represent some of the infinitesimal reasoning in Sir Isaac Newton's Philosophiæ Naturalis Principia Mathematica [17].

\footnotetext{
${ }^{\dagger}$ Incidentally, Isabelle is the program used in the project that this thesis describes; see Chapter 3
} 


\section{Chapter 2}

\section{The choice of an axiom system}

\subsection{Euclid's axioms}

When discussing the choice of an axiom system for which to demonstrate the independence of Euclid's parallels postulate, we must first address the question: Why should we not use Euclid's axioms?

By modern standards, Euclid was not careful enough in his application of the axiomatic method. A commonly cited fault is in the proof of his very first proposition in Book I (see [7, pages 241-242]); he implicitly assumes that if two circles each pass through the centre of the other, then they intersect; this is not justified by the axioms. Another example is that in the proof of I.16 (that is, Book I, Proposition 16; see [7, pages 279-280]), Euclid implicitly makes an assumption about betweenness; the elliptic plane satisfies all of Euclid's explicitly stated postulates, ${ }^{*}$ but in the elliptic plane, I.16 is false.

This being said, Euclid should not be denigrated too much. He was, after all, a very early pioneer of the axiomatic method, so it is not sur-

*However, if the parallels postulate is not replaced by, for example, Playfair's axiom, then the reader must do some semantic acrobatics to understand phrases like "on the same side". For example, the reader may wish to interpret statements like "The points $P$ and $Q$ are on the same side of the line $l^{\prime \prime}$ as tautologies. 
prising that his work is not perfect according to modern standards. To his credit, he shows great insight in the axioms that he chose to state explicitly. It has already been noted (see Section 1.3) that many highly capable mathematicians over about two thousand years believed that Euclid need not have stated the parallels postulate as an axiom; they were wrong, and Euclid was right to have explicitly stated it. Even Euclid's fourth postulate (about the equality of all right angles) is something that could easily have been implicitly assumed without justification by a less careful mathematician.

Having established that Euclid's axioms are not suitable for highly strict mechanical reasoning, we must now decide which axiom system to use. Many axiom systems are available; for example, the axiom systems used by H. S. M. Coxeter [5], by Karol Borsuk and Wanda Szmielew [3], or by F. Bachmann [1].

In addition, we have already discussed in Section 1.6 an axiom system adapted by Fleuriot from the work of Chou and his colleagues; this has the disadvantage that it is not categorical. If we can show the independence of the parallels postulate in a categorical axiom system, we can be sure that the parallels postulate is not implied by any other "missing" axioms that would more fully characterize geometry as we understand it.

Let us consider in more detail two axiom systems that I am aware have been used in mechanical proof verification. First, in Section 2.2, we consider David Hilbert's axioms, used in mechanical proof verification by Christophe Dehlinger, Jean-François Dufourd, and Pascal Schreck in [6], by Laura Meikle and Jacques Fleuriot in [15], and by Phil Scott in [22]; then, in Section 2.3, we consider Alfred Tarski's axioms, used by Julien Narboux in [16]. Section 2.4 lists and explains the axioms of the chosen system. 


\subsection{Hilbert's Grundlagen}

In 1899, Hilbert published the first edition of his now famous Grundlagen der Geometrie (available in English translation as [12]). In it he presented twenty-one axioms of geometry, proved some consequences of the axioms, and discussed some consistency and independence results about the axioms. His work greatly improved on Euclid's rigour, but Hilbert was not infallible, either.

Although Hilbert was trying to eschew reasoning justified only by intuition, he appears (for example) to have at least once made the unjustified assumption that a particular point in one of his proofs lay in a particular plane; in this case, Meikle and Fleuriot mechanically verified a corrected version of Hilbert's proof (see [15, page 327]), showing that Hilbert's intuition was correct that the point lay in the plane, but his reasoning was flawed.

For now, we are interested in whether Hilbert's axiom system is suitable for establishing the independence of his version of the parallels postulate; this is not the case. Hilbert's axioms require distinct primitive notions for points, lines, and planes, and many distinct primitive relations: point-line incidence, point-plane incidence, betweenness of points, congruence of line segments, and congruence of angles.

To establish the independence of the parallels postulate, we would have to define a model of all of Hilbert's axioms except the parallels postulate. Even if we restrict ourselves to the plane, our model would have to provide interpretations of points and lines, and definitions for each of the primitive relations except point-plane incidence; then we would have to prove that the model satisfies the large number of relevant axioms (and the negation of the parallels postulate). This is a daunting task. 


\subsection{Tarski's axioms}

As described in [29] and [16], Tarski's axioms for geometry have been refined and improved over time. His system was first described in lectures in 1926-1927, but not published until 1948 in [27, pages 55-57] (see [29, pages 188-189]). Incorporating many possible improvements that had been discovered by then, a more concise version of the axioms appeared in [21, pages 11-15] in 1983.

The axiom system uses just one primitive notion - that of points and two primitive relations - congruence of line segments, and betweenness.

For betweenness, $\mathrm{B} a b c$ can be understood as asserting that $a, b$, and $c$ are collinear and that $b$ lies between $a$ and $c$. This betweenness is not strict; if $b=a$ or $b=c$, then $\mathrm{B} a b c$ is true.

For congruence, $a b \equiv c d$ can be understood as asserting that the line segment with endpoints $a$ and $b$ is congruent to the line segment with endpoints $c$ and $d$. Alternatively, the reader may wish to interpret it as asserting that the distance from $a$ to $b$ is equal to the distance from $c$ to $d$.

Since we have noted in Section 2.2 Hilbert's flawed reasoning, it is only fair to point out that Narboux found some missing steps in the reasoning of Wolfram Schwabhäuser, Wanda Szmielew, and Alfred Tarski in [21] - see [16, pages 147-148]. However, as before, we are less interested in whether the axiom systems are always used perfectly by their authors, and more interested in whether they are useful for mechanically verifying the independence of the parallels postulate.

The eleven axioms adopted by Schwabhäuser and his colleagues in [21] are categorical (see [29, page 195]), satisfying one of the properties desirable for our purposes. In addition, one of the axioms (the tenth) is identified as the Euclidean axiom - the axiom equivalent to the parallels postulate. Also, there is a discussion in [29, pages 199-200] about the independence of many of the axioms (including the Euclidean axiom), 
assuring us of two things: first, in the case of those axioms known to be independent, we know that we are not performing unnecessary work when we verify that our model satisfies those axioms; second, we are assured that our goal of verifying the independence of the parallels postulate is not impossible. Finally, [29, pages 192-195] praises the relative simplicity of Tarski's axioms, giving us even more confidence that we will not be performing unnecessarily complicated verifications that a model satisfies the axioms.

For these reasons Tarski's axioms - as adopted in [21] — are suitable for this project's mechanical verification of the independence of the parallels postulate.

\subsection{Statement and explanation of Tarski's axioms}

The axioms are as follows. For each axiom, the name (adapted from its name in [29, pages 177-185]) is given first, then the formal statement of the axiom, and then a less precise, more intuitive explanation of the role of the axiom, to assist in understanding.

1. Reflexivity axiom for equidistance

$$
\forall a b . a b \equiv b a
$$

A line segment is congruent to its reverse.

2. Transitivity axiom for equidistance

$$
\forall a b p q r s . a b \equiv p q \wedge a b \equiv r s \longrightarrow p q \equiv r s
$$

If a line segment is congruent to two other line segments, then the latter are congruent to each other.

3. Identity axiom for equidistance

$$
\forall a b c . a b \equiv c c \longrightarrow a=b
$$




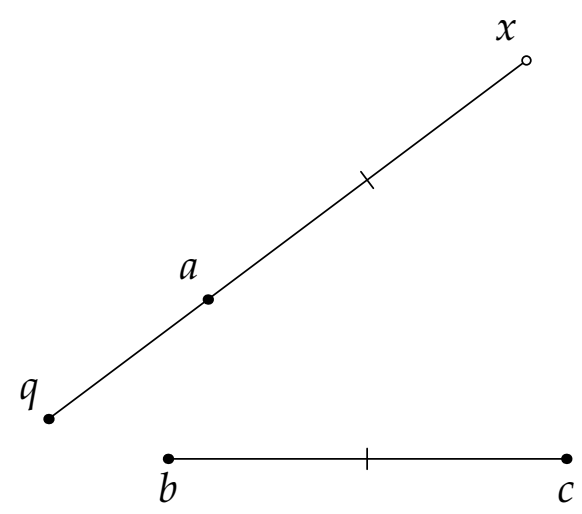

Figure 2.1: Tarski's axiom $4-$ the axiom of segment construction

If a line segment is congruent to a degenerate segment, then it must also be degenerate.

4. Axiom of segment construction (see Figure 2.1)

$$
\forall a b c q . \exists x . \text { В qax } \wedge a x \equiv b c
$$

Given a segment $(b c)$, a point (a), and a direction (that of $q a)$, we can construct a segment $(a x)$ congruent to the given segment, starting from the given point, and proceeding in the given direction.

5. Five-segments axiom (see Figure 2.2)

$$
\begin{aligned}
\forall a b c d a^{\prime} b^{\prime} c^{\prime} d^{\prime} . a \neq & b \wedge \mathrm{B} a b c \wedge \mathrm{B} a^{\prime} b^{\prime} c^{\prime} \\
& \wedge a b \equiv a^{\prime} b^{\prime} \wedge b c \equiv b^{\prime} c^{\prime} \wedge a d \equiv a^{\prime} d^{\prime} \wedge b d \equiv b^{\prime} d^{\prime} \\
\longrightarrow & c d \equiv c^{\prime} d^{\prime}
\end{aligned}
$$

Coxeter [5, page 180] usefully and succinctly describes a slight variant of this axiom as "ensuring the rigidity of a "triangle with tail"". In Figure 2.2, the triangle is $a b d$, and the "tail" is $b c$. More generally and less precisely, this axiom can be thought of as being responsible for ensuring that figures are not distorted when they are moved 


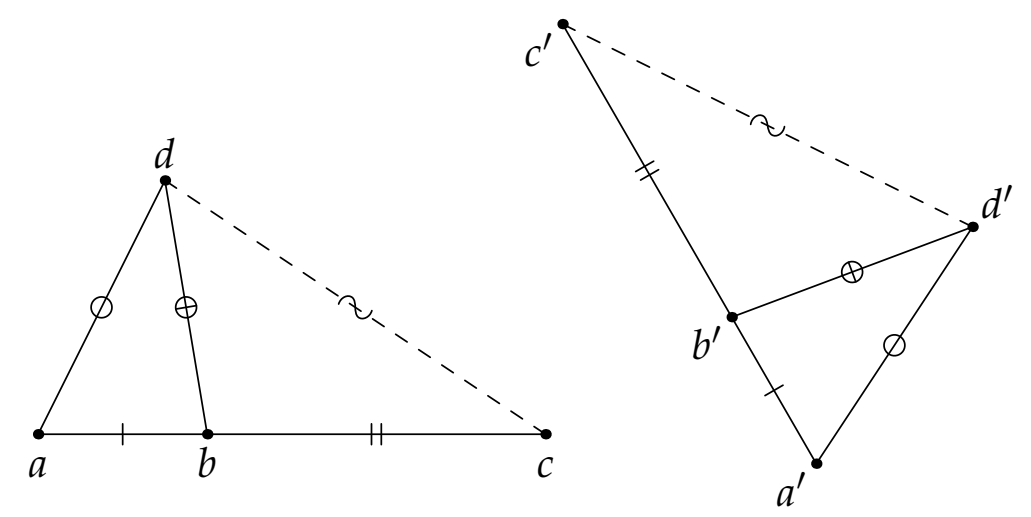

Figure 2.2: Tarski's axiom 5 - the five-segments axiom

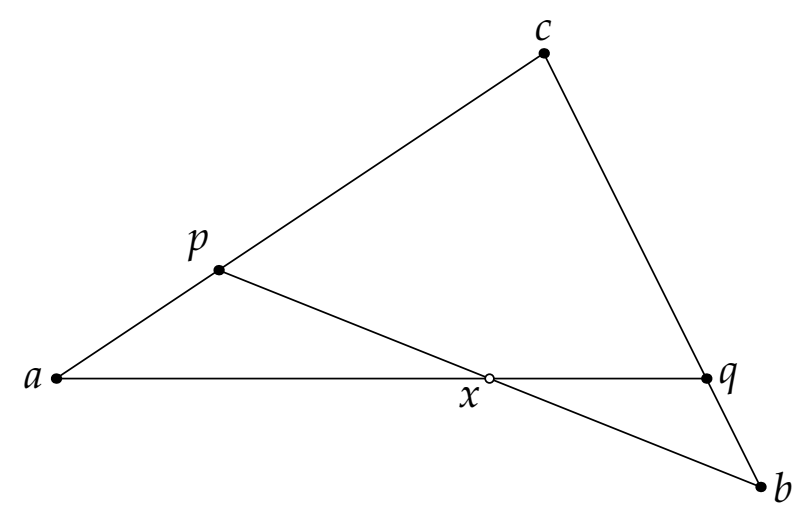

Figure 2.3: Tarski's axiom 7 - the axiom of Pasch

around by translations, reflections, and so on (but it does not guarantee that the plane can be dilated in a non-distorting way).

6. Identity axiom for betweenness

$$
\forall a b . \mathrm{B} a b a \longrightarrow a=b
$$

The only point between $a$ and $a$ is $a$ itself.

7. Axiom of Pasch (see Figure 2.3)

$\forall a b c p q . \mathrm{B} a p c \wedge \mathrm{B} b q c \longrightarrow \exists x . \mathrm{B} p x b \wedge \mathrm{B} q x a$ 
This axiom is a variant of one published by Moritz Pasch in [19, page 21]. Although the hypotheses of Tarski's version of the axiom are (in some ways) stricter, it can be understood as ensuring that if a line intersects one side of a triangle, it must also intersect another of the sides; this latter formulation is closer to Pasch's original axiom, and very similar to Hilbert's formulation (see [12, pages 4-5]).

In Figure 2.3, line $b p$ intersects side $a c$ of triangle $a c q$, so it must also intersect another of the sides; in this case, it must be side $a q$, since $b p$ intersects the line $c q$ outside the segment $c q .{ }^{+}$

8. Lower 2-dimensional axiom

$$
\exists a b c . \neg \mathrm{B} a b c \wedge \neg \mathrm{B} b c a \wedge \neg \mathrm{B} c a b
$$

There are three non-collinear points. This ensures that the geometry that this axiom system describes has at least 2 dimensions.

9. Upper 2-dimensional axiom (see Figure 2.4)

$\forall a b c p q . p \neq q \wedge a p \equiv a q \wedge b p \equiv b q \wedge c p \equiv c q \longrightarrow \mathrm{B} a b c \vee \mathrm{B} b c a \vee \mathrm{B} c a b$

The perpendicular bisector of two distinct points ( $p$ and $q$ in Figure 2.4 ) is a line. (More strictly, this axiom ensures only that the perpendicular bisector is a subset of a line, although we have not yet defined terms such as line.) This ensures that the geometry has at most 2 dimensions; in 3 dimensions, for example, the "perpendicular bisector" of two distinct points is a plane.

10. Euclidean axiom (see Figure 2.5)

$\forall a b c d t . \mathrm{B} a d t \wedge \mathrm{B} b d c \wedge a \neq d \longrightarrow \exists x y . \mathrm{B} a b x \wedge \mathrm{B} a c y \wedge \mathrm{B} x t y$

It is not immediately obvious how this axiom relates to Euclid's

\footnotetext{
${ }^{\dagger}$ Remember, this is intended as an explanation, not a proof; we have not proven that the line $b p$ cannot intersect the line $c q$ more than once; in fact, it does so in the special case where $p=c$.
} 


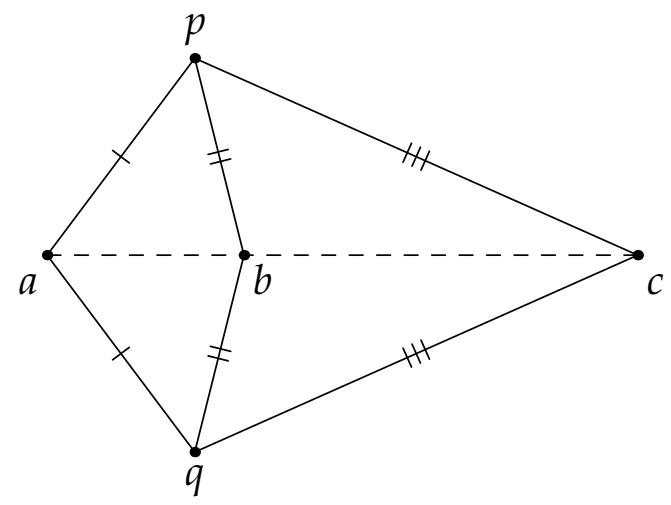

Figure 2.4: Tarski's axiom 9 - the upper 2-dimensional axiom

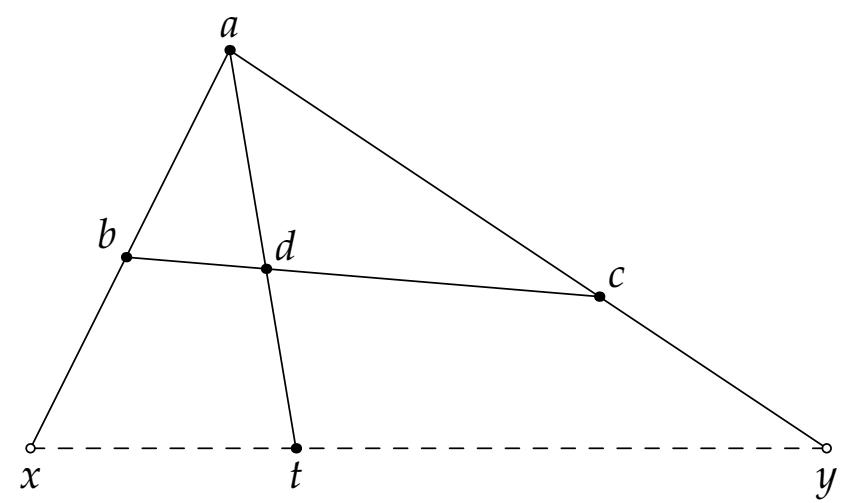

Figure 2.5: Tarski's axiom 10 - the Euclidean axiom 


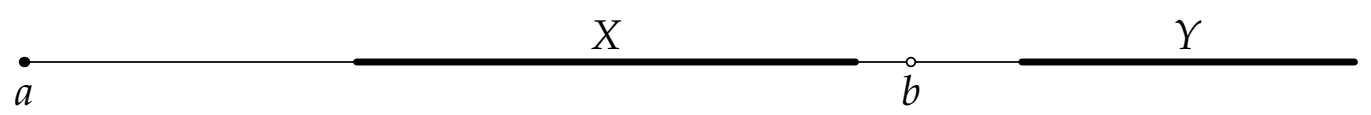

Figure 2.6: Tarski's axiom 11 - the axiom of continuity

fifth postulate or to Playfair's axiom. Without going into too much detail, it may be beneficial to observe how this axiom succeeds in Euclidean geometry in a general case, and how it fails in hyperbolic geometry.

In Euclidean geometry, suppose we are given $a, b, c, d$, and $t$ such that the hypotheses of the axiom hold. For non-degeneracy of this explanation, assume also that $a, b$, and $d$ are not collinear. Then we can construct the line $x y$ parallel to $b c$. That is, we can draw a line through $t$ that makes the same angle with the line at as the line $b c$ makes with at; this new line is, in fact, parallel to $b c$, and, more importantly for the truth of the axiom, it intersects the lines $a b$ and $a c$; we take these intersection points as our choices of $x$ and $y$, respectively.

In the hyperbolic case, suppose again that we are given $a, b, c, d$, and $t$ as before, and that in addition, $c \neq d$. We may again draw a line through $t$ making the same angle with $a t$ as $b c$ makes with at. However, it may be the case that $t$ is so far from $a$ that this newly constructed line fails to intersect lines $a b$ and $a c$. Pivoting the new line around $t$ will allow us to ensure that it intersects either line $a b$ or line $a c$ at an appropriate point, but it cannot do both.

11. Axiom of continuity (see Figure 2.6)

$$
\begin{aligned}
& \forall X Y .(\exists a . \forall x y . x \in X \wedge y \in Y \longrightarrow \mathrm{B} a x y) \\
& \longrightarrow(\exists b . \forall x y . x \in X \wedge y \in Y \longrightarrow \mathrm{B} x b y)
\end{aligned}
$$

The first thing to note about this axiom is that it is not a first-order 
axiom; the variables $X$ and $Y$ range over sets of points rather than over points themselves. If we wanted a purely first-order axiom system, we could replace this axiom with an axiom schema; that is, we could instead adopt every axiom of the form

$$
(\exists a . \forall x y \cdot \alpha \wedge \beta \longrightarrow \mathrm{B} a x y) \longrightarrow(\exists b . \forall x y \cdot \alpha \wedge \beta \longrightarrow \mathrm{B} x b y)
$$

where $\alpha$ and $\beta$ are first-order formulas such that $\alpha$ has no free occurrences of $a, b$, or $y$, and $\beta$ has no free occurrences of $a, b$, or $x$ (see [29, page 185]). In our case, this is neither necessary nor desirable: it is unnecessary because there are proof verification programs that work well with higher-order logic; and it is undesirable because a purely first-order axiom system for geometry would be susceptible to the Löwenheim-Skolem theorem, which would imply that it could not possibly be categorical. For these reasons, we adopt the higher-order version of this axiom.

This axiom has some of the flavour of the Dedekind-cuts construction of the real numbers, or of the theorem that a non-empty set of reals must have an infimum if it is bounded below (see [29, pages 198-199]). To aid in understanding it, let us examine how this axiom rules out the rational Cartesian plane $\mathbb{Q}^{2}$ as a model of the system of axioms. $\ddagger$

Take $X=\left\{(p, 0) \mid p>0 \wedge p^{2}<2\right\}$ and $Y=\left\{(p, 0) \mid p>0 \wedge p^{2}>\right.$ $2\}$. Take $a=(0,0)$. Then it is certainly the case (with the natural definition of betweenness for this "model") that for each $x \in X$ and each $y \in Y$, we have B axy. However, our only possible choice for $b$ is $(\sqrt{2}, 0)$, which is not present in $\mathbf{Q}^{2}$.

\footnotetext{
$\ddagger$ In fact, axiom 4 - the segment construction axiom - also rules out $\mathbb{Q}^{2}$ as a model. To establish that axiom 11 is independent, we must replace $\mathbb{Q}$ with a Pythagorean ordered field not isomorphic to $\mathbb{R}$ (see [21, pages 16-17]); the hyperreals form such a field, but pursuing this in detail is beyond the scope of this work.
} 


\section{Chapter 3}

\section{The choice of a proof verification program}

\subsection{Wiedijk's Seventeen Provers}

When a mathematician or computer scientist is choosing a proof verification program, one excellent resource is Freek Wiedijk's The Seventeen Provers of the World [33]. It is a comparison of the features of seventeen worthy proof verification programs. The bulk of the text is provided by users of the systems, each of whom was asked to demonstrate a proof of the irrationality of $\sqrt{2}$ in their system. Users were also asked some other questions about the systems, such as "What are the books about the system?", and "What is the logic of the system?".

Wiedijk himself provides a particularly useful table of features, showing which proof verification programs have which features - see [33, page 11]. In Section 3.2 we consider some of the features that are essential for reliable proof verification programs; in Section 3.3 we consider features that are not essential, but would be useful for a broad range of proof verification work; and in Section 3.4 we consider features that are particularly relevant to this project's verification of the independence of the parallels postulate. 
Section 3.5 concludes this chapter by describing the proof verification program that was chosen for this project.

\subsection{Essential features}

The first feature in Wiedijk's table of features is a "small proof kernel". The idea behind this is that a small part (the "kernel") of the proof verification program checks everything that the software verifies. This way, if the software incorrectly validates a proof, there must be an error within the kernel; contrapositively, if there is no error in the kernel, then every proof that the software validates must indeed be valid.

Suppose a user wishes to convince themself that the software only allows valid proofs; then they need only check the correctness of the kernel.

If proof verification software lacks a small kernel, then doubt may be cast on the validity of its judgements. A bug may easily remain hidden in thousands of lines of source code, and without a separate kernel, a bug anywhere may be fatal to the soundness of the system as a whole.

On the other hand, if the software has a small kernel, programmers may freely add features outside the kernel - such as automated calculation and proof search - safe in the knowledge that even if their additions contain bugs, these bugs will not affect the soundness of the whole program.

Related to this is another important feature not mentioned in Wiedijk's table. In order to be sure about the correctness of a proof verification program, the user must have access to its source code; without the source code, the task of checking the correctness of the kernel is made unnecessarily difficult, if not impossible without the assistance of automated machine-code analysis (which would raise questions about the program used to analyse the machine code).

Most of the most popular proof verification programs are free, open 
source software, including HOL Light, Coq, and Isabelle. However, at least one of the proof verification programs discussed in [33] does not have publicly available source code — specifically, Mizar (see [31]).

\subsection{Desirable features}

One feature that is desirable in any proof verification program is a large body of proofs already verified by the program (called a "large mathematical standard library" in Wiedijk's table). Users of the program can then refer to already proven theorems when they use them in their own proofs, without having to prove everything from scratch.

Another desirable feature is that the input files written by mathematicians are readable. This is perhaps best illustrated by example.

The following is the proof in HOL Light of the irrationality of $\sqrt{2}$ given in [33, page 18] (after necessary definitions and lemmas). Line-breaks are adjusted to fit the present margins.

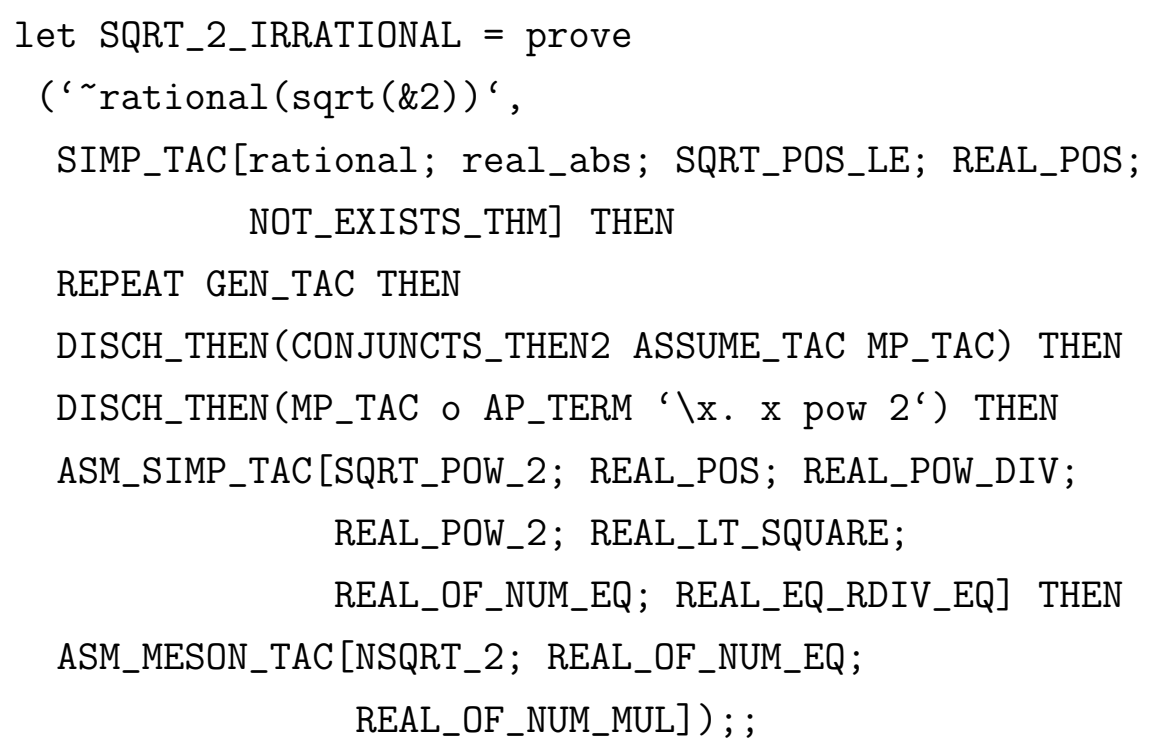

In contrast, here is an excerpt from the Mizar proof, taken from [33, page 28]. 


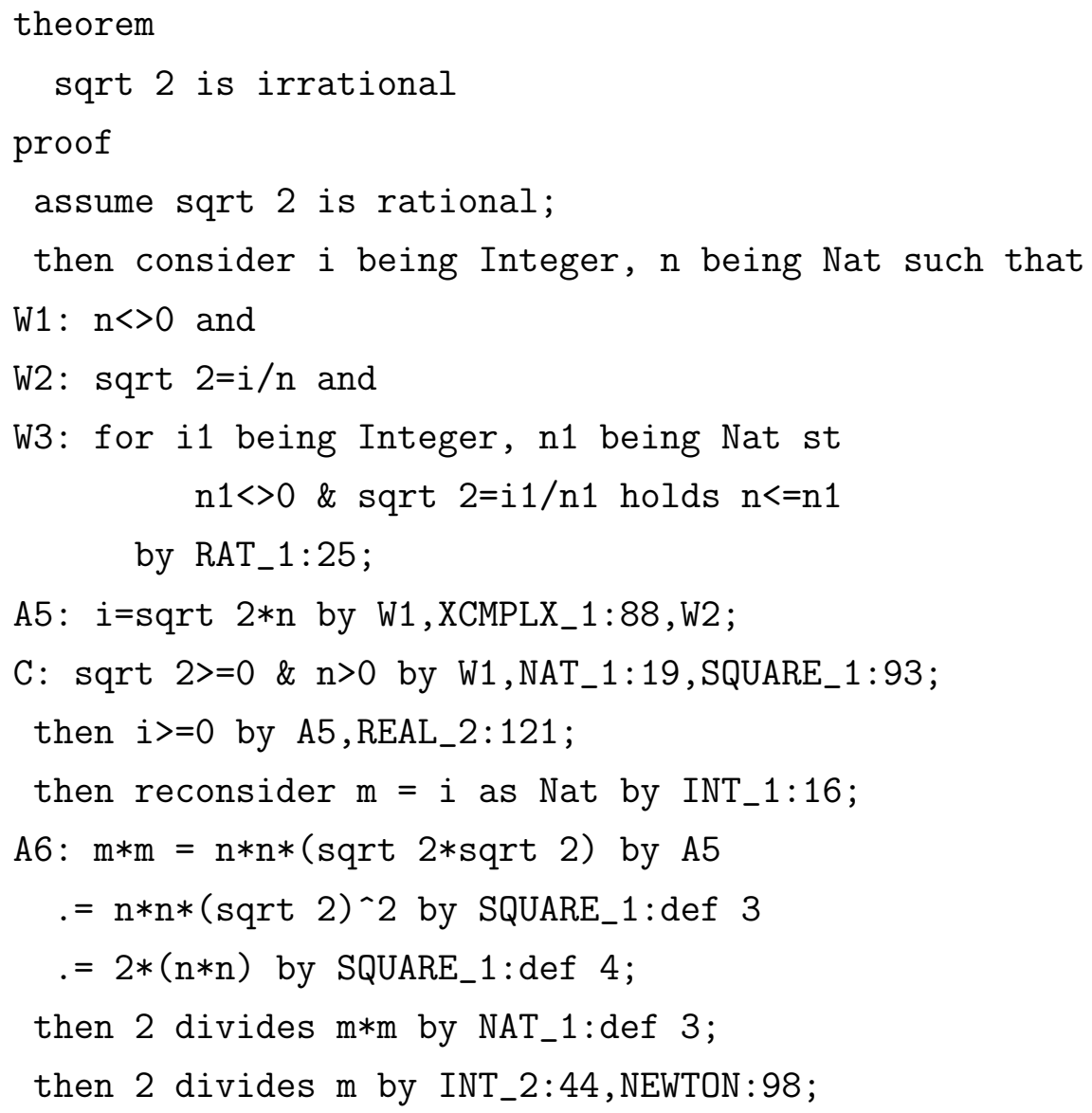

A mathematician who has no experience with Mizar has a good chance of being able to understand the nature of this proof.

The HOL Light proof, on the other hand, is quite opaque. There is a danger that a proof that is verified by HOL Light is subtly (or even significantly) different from the proof in the readers' (or even the authors') minds, denying these mathematicians insights into the true nature of the proof; the proof in the mathematicians' minds may even be logically invalid, even if HOL Light has found a valid proof.

Apart from ensuring that a proof script is indicative of the nature of the proof, human-readability of input files serves another purpose: aiding the maintenance of proof scripts. If, for example, SIMP_TAC becomes more powerful in a future version of HOL Light, it may cause the internal 
state of the proof after that line to differ depending on the version of HOL Light used; subsequent commands in the proof shown above may then fail because the proof state is different from that which the author of the proof expected. To fix this, a proof maintainer may need to try to comprehend the original proof and reconstruct it using a different sequence of commands.

Compare this scenario with a similar one in Mizar. Suppose that, in a future version of Mizar, REAL_2:121 is altered in a way that causes that line of the above proof to fail. Then a proof maintainer can quickly see that from $\sqrt{2} \geq 0, n>0$, and $i=\sqrt{2} n$, the author of the proof deduced that $i \geq 0$; the maintainer can then replace REAL_2:121 with the appropriate theorem or rule of inference, and the rest of the proof is unaffected and need not be revisited.

\subsection{Features useful for this project}

With the particular purpose in mind of establishing the independence of the Euclidean axiom within Tarski's axiom system for plane geometry, we can consider which features of various proof verification programs might be useful for this project.

One useful feature would be a pre-existing formalized theory of the real numbers. Without this, we would essentially need to construct our own model of $\mathbb{R}$ in order to establish the consistency of Tarski's axioms of plane geometry, because $\mathbb{R}^{2}$ is the only model (up to isomorphism) of Tarski's axioms. In particular, the greatest-lower-bound property of the real numbers would be a major asset in proving that a model of Tarski's axioms satisfies the axiom of continuity.

The real numbers will certainly also be useful in establishing the independence result, as well as the consistency result discussed in the previous paragraph. For this reason, we desire a proof verification program with pre-defined real numbers, and suitable theorems about great- 
est lower bounds.

As discussed in Section 2.4, we wish to use the higher-order version of the axiom of continuity. In order to do so without undue hassle, we need a proof verification program that supports higher-order logic. Fortunately, there are several such proof verification programs, and most of them support typed higher-order logic.

In such systems, there are many different types, such as a type of natural numbers, a type of real numbers (if the system supports the real numbers), a type of functions from $\mathbb{R}$ to $\mathbb{R}$, and a type of sets of real numbers. This ensures that users of the system cannot inadvertently form propositions about whether a real number is equal to the cosine function, for example; the system would complain about type correctness. This safety is impossible in first-order Zermelo-Fraenkel set theory, because $\sqrt{3}$ (considered as a real number) and the cosine function are both simply sets, and as far as the logic is concerned, they may or may not be equal to each other.

Of course, when a mathematician proves that a certain structure is isomorphic to a substructure of a larger structure that was defined later, they often wish to identify the earlier structure with the isomorphic substructure of the later structure; for example, mathematicians often treat natural numbers as if they are integers, even if they have defined integers to be equivalence classes of ordered pairs of natural numbers. Accommodating this is difficult in any highly formalized system, but it is easy to provide users of the system with suitable injection functions, including, for example, a function from the type of natural numbers to the type of integers.

\subsection{Isabelle}

Considering all of the essential and desirable features mentioned in Sections 3.2-3.4, one proof verification program stands out as being the most 
suitable: Isabelle. Indeed, according to Wiedijk's table (which is, admittedly, several years old), Isabelle is the only system that has both a small proof kernel and a system allowing human-readable input files.

In fact, the only desirable feature that Isabelle is noted as lacking in Wiedijk's table is "dependent types". Isabelle does allow the definition of types that depend on other types - for example, product types and function types. However, it does not allow the definition of types that depend on elements of other types. Such a feature would be useful in defining quotient rings, for example.

This defect may be partially mitigated by the "quotient type" feature introduced in Isabelle 2009-2 in June 2010. However, this project to mechanically verify the independence of the parallels postulate was started in early 2009 using Isabelle 2008; although the project was subsequently altered to work with Isabelle 2009-2 (but not yet with any later version of Isabelle), the parts that might have benefited from quotient types had already been written, and adjusting them to make use of quotient types would have created unnecessary work.

One particularly useful feature of Isabelle is its locales. Locales allow the user to develop axiomatic theories, such as the theory of metric spaces. The user could define a locale for metric spaces, giving the axioms of metric spaces. Then the user is able to prove consequences of those axioms, and provide models for the axioms (with proof).

The major benefit of this is that once the user has proven that real vector spaces, for example, satisfy the axioms of a metric space, then they may immediately use the theorems that they have proven about metric spaces, without having to re-prove them in the specific case of real vector spaces.

As is mentioned in Section 5.2, a very brief version of this programme regarding metric spaces was carried out for this project. 


\section{Chapter 4}

\section{Locales}

\subsection{Overview}

Isabelle's locales can build on previously defined locales, so instead of only a single locale being used for Tarski's axioms, the axioms were broken up into groups for various reasons.

Section 4.2 describes the locale that was defined in Isabelle to formalize the first three of Tarski's axioms; this section covers the locale in some detail, to familiarize the reader with Isabelle's notation. Then, Section 4.3 covers the locale that formalizes Tarski's first five axioms. With the first five axioms formalized, Section 4.4 explains the formalization of a simple theorem that is a consequence of those axioms; using this theorem as an example, Section 4.5 illustrates the benefit of using many incremental locales to formalize Tarski's axioms, instead of just one locale. Section 4.6 then describes the formalization of Tarski's remaining axioms.

The formalization that was written for this project is very long - far too long to include in this thesis. However, this and subsequent chapters contain a number of excerpts from the formalization; these were typeset using Isabelle's automatic typesetting facility. A typeset version of the entire formalization is available as [14]. 


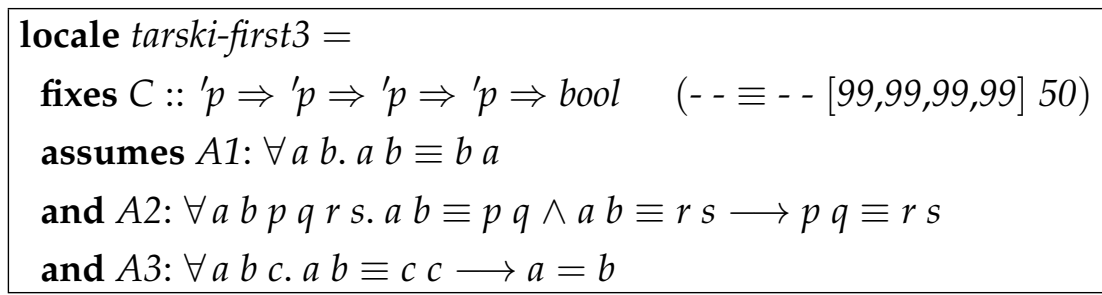

Figure 4.1: Tarski's first three axioms formalized in Isabelle

\subsection{A locale for Tarski's first three axioms}

Tarski's first three axioms use only congruence, not betweenness, so a locale was defined for those axioms; users need not define a betweenness relation for a structure that they wish to prove is a model of those axioms. The locale was defined as in Figure 4.1.

In the first line, the locale is named tarski-first3, for future reference.

In the second line, a function $C$ is fixed, to act as the four-place relation of congruence on the type ' $p$; this type represents the type of points of the geometry. Internally, the four-place relation is represented by a function from the type ${ }^{\prime} p$ to the type ${ }^{\prime} p \Rightarrow{ }^{\prime} p \Rightarrow{ }^{\prime} p \Rightarrow b o o l$; this latter type is the type of functions from ${ }^{\prime} p$ to ${ }^{\prime} p \Rightarrow{ }^{\prime} p \Rightarrow b o o l$, and so on; bool is a Boolean type whose only elements are True and False. The end of the second line establishes an optional notation for the congruence relation, so that we can write the first axiom, for example, as $\forall a b . a b \equiv b a$ instead of $\forall a b$. $C a b b a$.

The final three lines in Figure 4.1 state the axioms, which are given labels $A 1, A 2$, and $A 3$, for future reference.

\subsection{A locale for Tarski's first five axioms}

Figure 4.2 shows the next locale, tarski-first5, whose first line causes it to inherit the relation $C$ and the axioms of tarski-first3. This locale fixes the betweenness relation $B$, but it stops short of listing all of Tarski's remain- 


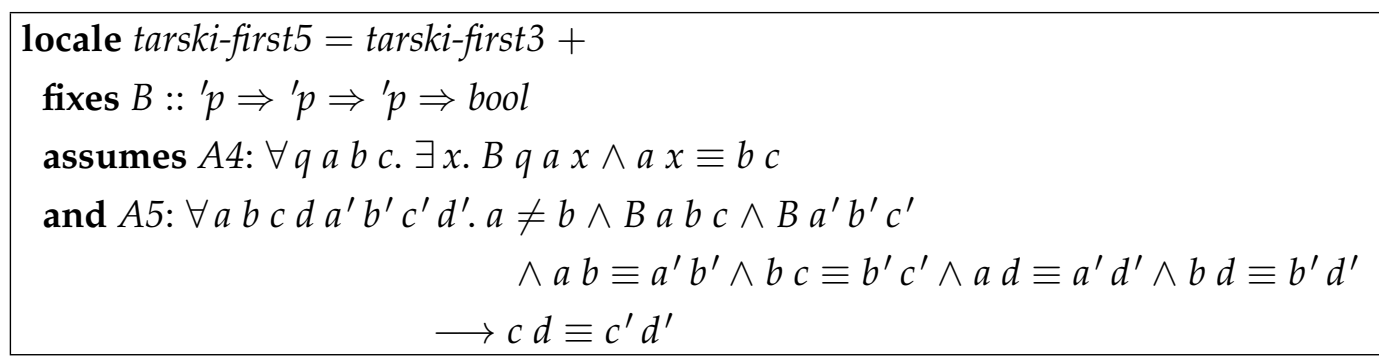

Figure 4.2: A locale for Tarski's first five axioms

theorem (in tarski-first5) th3-1: $B a b b$

proof -

from $A 4[$ rule-format, of $a b b b]$ obtain $x$ where $B a b x$ and $b x \equiv b b$ by auto

from $A 3$ [rule-format, of $b x b]$ and $\langle b x \equiv b b\rangle$ have $b=x$ by simp

with $\langle B a b x\rangle$ show $B a b b$ by simp

qed

Figure 4.3: A short proof in Isabelle

ing axioms. This is because there are some results that can be proven from only the first five axioms; by using locales with only the necessary axioms, we obtain a stronger result than if we had written proofs with all of Tarski's axioms as initial assumptions.

In fact, Schwabhäuser and his colleagues wrote a short chapter (see [21, pages 27-29]) on some of the consequences of the first five axioms. Some of these results were formalized in Isabelle (following quite closely the proofs - and even theorem numbering - in [21]); see [14, pages 16-22].

\subsection{A simple proof in a locale}

Although Schwabhäuser and his colleagues left it until the next chapter, their Satz 3.1 (see [21, page 30]) is also a consequence of the first five axioms; this fact is verified in Isabelle, and shown in Figure 4.3. Isabelle's 
human-readable language makes this relatively easy to follow, but a few notes may be beneficial.

The first line ensures that the theorem is proven (and can later be used) in the context of tarski-first5; it also names the theorem th3-1 and gives the statement: $B a b b$. Notice that the variables are not explicitly quantified; when using this theorem, the variables can be instantiated by any elements of the correct type.

The axioms $A 3$ and $A 4$ are now treated as ordinary theorems, but are manipulated for use here. First, rule-format instructs Isabelle to attempt to derive a version of the theorem in the form of an inference rule. For example, $A 3$ [rule-format $]$ means $? a ? b \equiv ? c ? c \Longrightarrow ? a=? b$. The schematic variables (preceded by question marks) are then instantiated by listing the desired instantiations in order after of; so, A3 [rule-format, of $b \times b]$ means $b x \equiv b b \Longrightarrow b=x$.

The words simp and auto in Figure 4.3 are the names of methods designed to find simple proofs from the given facts and some standard simplification theorems; the proofs are then checked by the Isabelle kernel. There are ways of manipulating which facts and theorems are made available to simp and auto, and there are other methods used sometimes in this project, including blast and fast.

Many of the methods, including simp and auto, can often provide the necessary instantiations of variables in the theorems that they use; the author of the proof can manually provide the instantiations if the automatic methods fail, or if they take too long to find the correct instantiations.

\subsection{The benefit of many locales}

Later in the formalization, when it had been established that $\mathbb{R}^{2}$ is a model of tarski-first5, it was convenient to have th3-1 already proven; this theorem was used to dispose of a degenerate case in the proof of a lemma called Col-dep2; this lemma was, in turn, used in the proof that $\mathbb{R}^{2}$ is a 


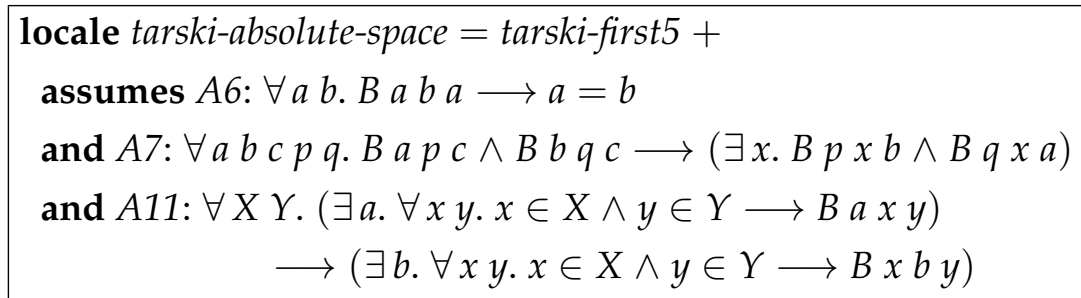

Figure 4.4: A locale for Tarski's axioms of absolute geometry

model of the lower and upper 2-dimensional axioms. This illustrates the usefulness of many incremental locales; with only one monolithic locale for Tarski's axioms of the Euclidean plane, the lower and upper 2-dimensional axioms would need to have been verified before th3-1 was able to be applied to $\mathbb{R}^{2}$, so the essence of the proof of th3-1 would need to have been repeated, unless another proof was found to verify those later axioms.

Similarly, Satz 2.2 from [21, page 27] (th2-2 in Isabelle) is (and was verified to be) a theorem in tarski-first3 (see [14, page 17]). Then, after our model of the hyperbolic plane was shown to satisfy tarski-first3, th22 was used in the proof that the model satisfies the axiom of segment construction (see [14, pages 179-181]).

\subsection{Locales for the remaining axioms}

On top of tarski-first5, we now add the remainder of Tarski's axioms of the absolute geometry of ( $n$-dimensional) space - encompassing both the Euclidean and the hyperbolic cases, and having no restriction on dimension. Figure 4.4 shows the locale defined for this purpose.

For some purposes, it may be beneficial to break this locale down even further; for instance, the axiom of continuity could be deferred in order to investigate the independence of that axiom, and to rigorously demonstrate which theorems do and do not rely on it. For this project, it was not found necessary to have such a fine-grained locale structure. 


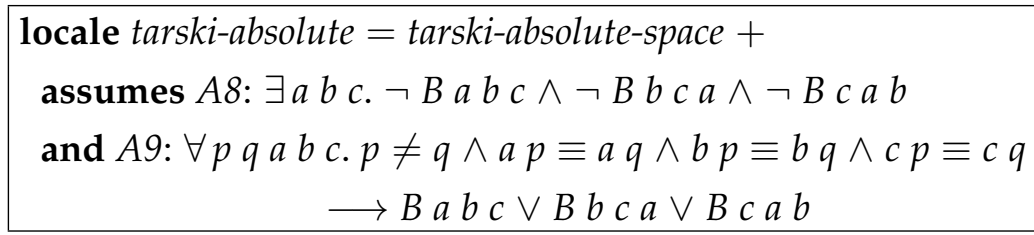

Figure 4.5: A locale for Tarski's axioms of absolute plane geometry

locale tarski-space $=$ tarski-absolute-space + assumes $A 10: \forall a b c d$ t. $B$ ad $t \wedge B b d c \wedge a \neq d$ $\longrightarrow(\exists x y \cdot B a b x \wedge B a c y \wedge B x t y)$

Figure 4.6: A locale for Tarski's axioms of Euclidean space

locale tarski $=$ tarski-absolute + tarski-space

Figure 4.7: A locale for Tarski's axioms of the Euclidean plane

Next, the dimension-specific axioms are added in another locale see Figure 4.5.

Unlike Euclid's original parallels postulate, or some forms of Playfair's axiom, Tarski's Euclidean axiom unequivocally carries the intended meaning regardless of how many dimensions the geometry has. Therefore, we can specialize tarski-absolute-space by adding only the Euclidean axiom, leaving out the dimension-specific axioms of tarski-absolute - see Figure 4.6.

Finally, having stated all the axioms in various locales, we can define a locale for all of Tarski's axioms of plane Euclidean geometry simply by unifying tarski-absolute and tarski-space - see Figure 4.7. 


\section{Chapter 5}

\section{The consistency of Tarski's axioms}

\subsection{The model}

Before moving on to the main goal of establishing the independence of Tarski's Euclidean axiom, it is worthwhile first verifying the consistency of Tarski's axioms; after all, if the axioms are inconsistent, then either Tarski's Euclidean axiom not only can, but must be false, or the inconsistency lies within Tarski's other ten axioms, and any question of the independence of the Euclidean axiom is necessarily meaningless.

In order to verify the consistency of Tarski's axioms, the easiest method is to provide a model of the axioms and prove that it is a model. As was mentioned in Section 3.4, every model of Tarski's axioms is isomorphic to $\mathbb{R}^{2}$. Isabelle already has a type defined for $\mathbb{R}$ and a two-element type 2; it also has a type constructor for Cartesian products of types; therefore, it is relatively easy to construct the appropriate model.

In fact, the model was defined for $\mathbb{R}^{n}$, and was proven to be a model of tarski-space in this more general case. Only for the two dimension-specific axioms was it necessary to write proofs specific to $\mathbb{R}^{2}$.

The Isabelle definitions of the relations intended to interpret $B$ and $C$ 


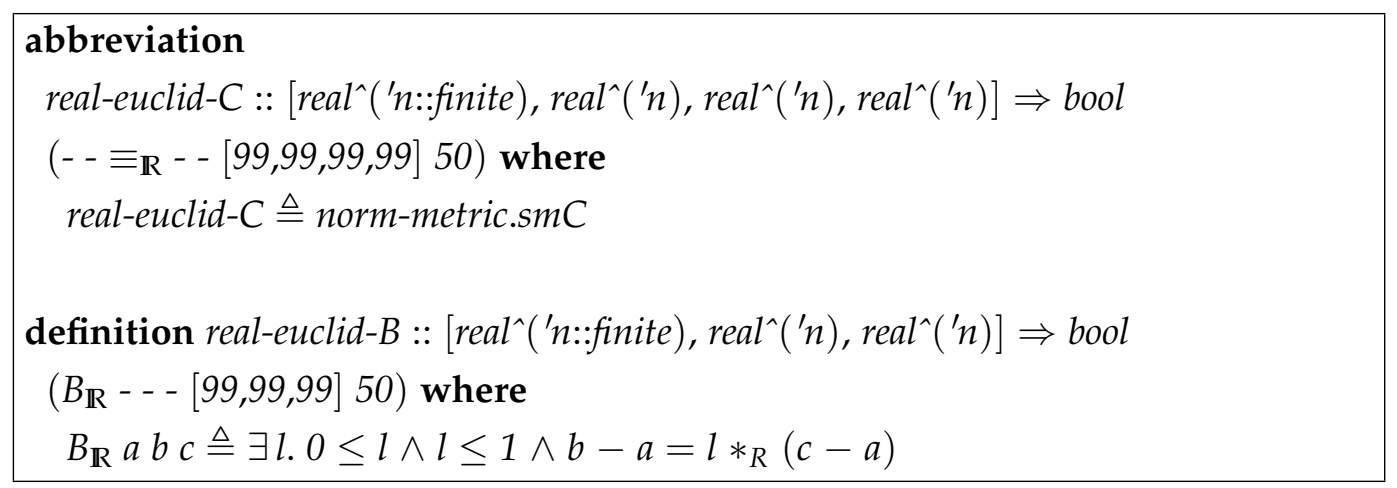

Figure 5.1: Definitions of congruence and betweenness in $\mathbb{R}^{n}$

are shown in Figure 5.1.

The definition of congruence is actually an abbreviation for a definition given in a more general context; this is covered in the next section. The difference between definitions and abbreviations in Isabelle primarily relates to whether or not they are automatically unfolded in proofs.

Betweenness for real vectors is called in Isabelle real-euclid-B (but given optional notation). The type of the relation, in the first line of the definition, is an abbreviation for the longer form analogous to the type of $B$ in the definition of the tarski-first5 locale in Figure 4.2. Notice that the type variable ' $p$ will be instantiated by real ${ }^{\wedge} n$. The type variable ' $n$ is constrained to be of a particular sort called finite; this guarantees that there are only finitely many elements of type ' $n$.

The definition of betweenness ensures that $B_{\mathbb{R}} a b c$ is true if and only if the vector representing $b$ is a convex combination of the vectors representing $a$ and $c$. 


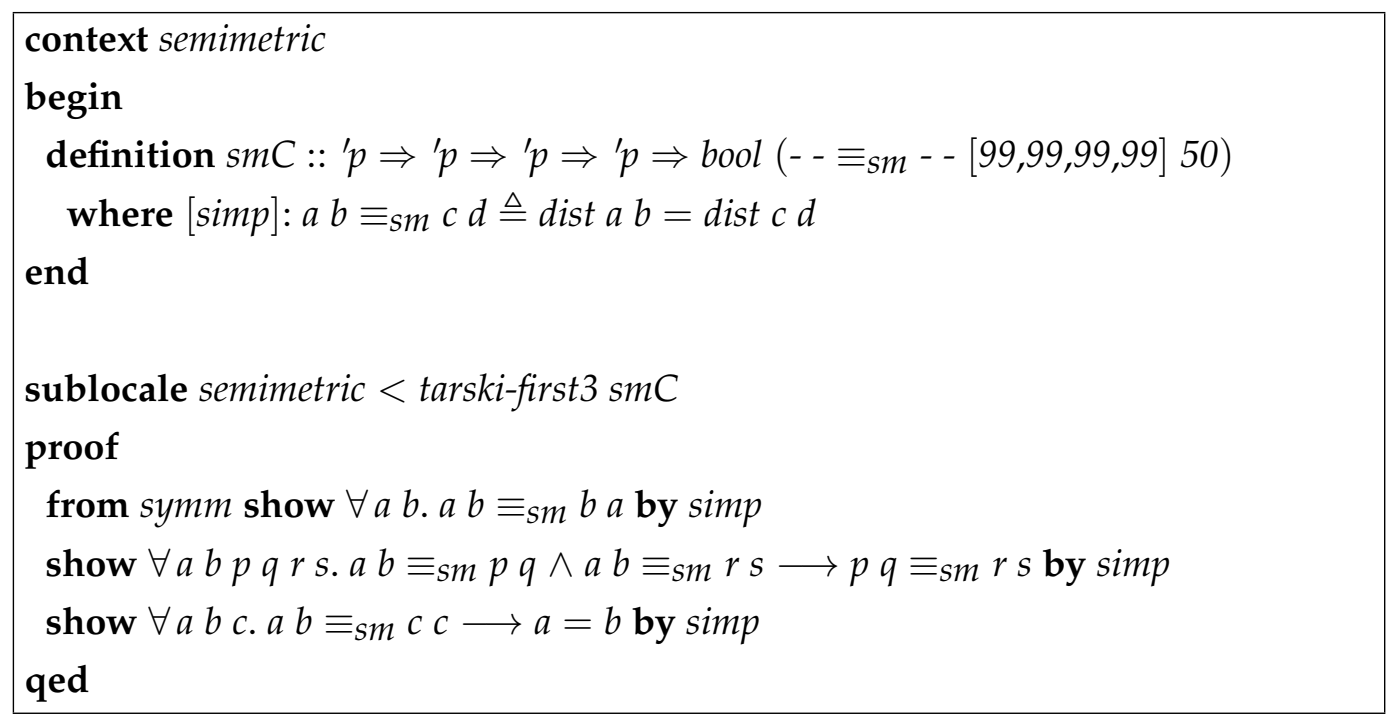

Figure 5.2: Semimetric spaces satisfy Tarski's first three axioms

\subsection{Congruence in semimetric spaces}

Metric and semimetric* spaces were defined in Isabelle for this project, and it was shown that a normed space is a metric space and that a metric space is a semimetric space (see [14, pages 2-3]); normed spaces had already been defined in Isabelle, and real vector spaces had been shown to be normed spaces.

Figure 5.2 shows how congruence was defined on an arbitrary semimetric space, and how it was shown to satisfy Tarski's first three axioms. The definition of congruence for semimetric spaces is declared to be part of the simpset - the set of theorems that can be automatically used by simp and auto when these methods are used to prove other theorems.

Because congruence on real vector spaces is defined as a special case of semimetric congruence, Isabelle immediately knows that it satisfies Tarski's first three axioms.

${ }^{*}$ A semimetric space is like a metric space: the distance function must be nonnegative, it must be 0 if and only if its arguments are equal, and it must be symmetric; however, it need not satisfy the triangle inequality. 
The proofs that real congruence and betweenness satisfy Tarski's other axioms vary in complexity. Some of the more interesting proofs are described in the remaining sections of this chapter; other proofs are omitted from this thesis, but the interested reader can refer to [14, pages 24-39].

\subsection{The five-segments axiom}

Suppose the five-segments axiom is considered as a theorem requiring proof, and suppose that the mathematician asked to prove it is familiar with Euclid's Elements and the style of proofs in that work. This mathematician might come up with a proof somewhat like the following:

By side-side-side congruence of triangles, we have triangle bad congruent to triangle $b^{\prime} a^{\prime} d^{\prime}$; therefore angle $b a d$ is equal to angle $b^{\prime} a^{\prime} d^{\prime}$. Because $a b \equiv a^{\prime} b^{\prime}, b c \equiv b^{\prime} c^{\prime}, \mathrm{B} a b c$, and $\mathrm{B} a^{\prime} b^{\prime} c^{\prime}$, we must have $c a \equiv c^{\prime} a^{\prime}$. By side-angle-side congruence of triangles, we have triangle cad congruent to triangle $c^{\prime} a^{\prime} d^{\prime}$; therefore $c d \equiv c^{\prime} d^{\prime}$, as required.

It may seem tempting to formalize this simple proof in Isabelle, to establish that our model does indeed satisfy the five-segments axiom. However, to do so would necessitate first formalizing the notion of angles in our model, and then proving theorems about the congruence of angles and triangles. It is desirable to find a simpler proof to formalize.

The cosine rule states that in a triangle whose sides are the vectors $u$, $v$, and $w$,

$$
\|w\|^{2}=\|u\|^{2}+\|v\|^{2}-2\|u\|\|v\| \cos \theta
$$

where $\theta$ is the angle between $u$ and $v$, which both emanate from their common vertex. At first, this may seem to complicate things more, by requiring not only angles to be formalized, but also the cosine function. Fortunately, it is also the case that $u \cdot v=\|u\|\|v\| \cos \theta$; this suggests a substitution that results in an easily proven vector identity: $\|w\|^{2}=$ 


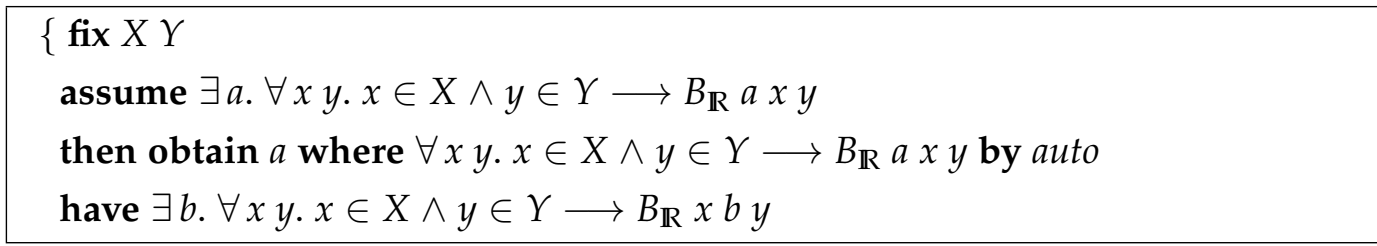

Figure 5.3: Beginning to verify the continuity axiom

$\|u\|^{2}+\|v\|^{2}-2 u \cdot v$ (think about what it means for $u, v$, and $w$ to be the sides of a triangle). This allows us to effectively use a function of an angle (its cosine) without having to define angles; the informal proof given at the start of this section can be adjusted to avoid any mention of angles.

The above strategy was used in the Isabelle verification that real Cartesian space satisfies the five-segments axiom (see [14, pages 26-29]). The proof is similar to an argument given by Henry George Forder in [10, page 201]; I did not discover Forder's argument until after writing the Isabelle formalization, otherwise the formalization might have been simpler.

\subsection{The axiom of continuity}

A mathematician writing a human-readable proof that $\mathbb{R}^{n}$ satisfies the axiom of continuity might begin by

- fixing the sets $X$ and $Y$,

- assuming the hypothesis $\exists a . \forall x y . x \in X \wedge y \in Y \longrightarrow \mathrm{B} a x y$,

- choosing such an $a$, and

- trying to prove the conclusion $\exists b . \forall x y . x \in X \wedge y \in Y \longrightarrow \mathrm{B} x b y$ by constructing such a $b$.

This is exactly how the formalized Isabelle proof begins - see Figure 5.3.

This axiom, as Figure 2.6 suggests, is primarily about the behaviour of points and sets of points that are constrained to a line. However, in order 
to carry out this proof rigorously, we must consider some degenerate cases. The most obvious degenerate cases occur when $X$ or $Y$ is empty; in these cases, the hypothesis and conclusion are both vacuously true, even if $Y$ or $X$, respectively, is the set of all points.

There is another, less obvious degenerate case. If $X$ has only a single element, then $a$ may have been chosen to be that element, and $Y$ may again be any set of points; Baay is always true. In this case, $b$ can be chosen to be $a$, and the conclusion is easily verified.

It was only after starting and struggling with the formalized proof of the non-degenerate case that I noticed the existence of this last degenerate case. This illustrates one of the benefits of writing computer-verifiable proofs: because the computer applies only the logic that it is explicitly allowed to apply, the mathematician who writes the proof is forced to do the same; no invalid logical steps can be made, and no special cases can be left unconsidered.

Figure 5.4 picks up where Figure 5.3 left off; it shows how all of the degenerate cases were considered at once in Isabelle, and also shows the start of the formalized proof in the non-degenerate case. The variable ? thesis refers to the goal currently requiring proof - in this case $\exists b . \forall x y$. $x \in X \wedge y \in Y \longrightarrow B_{\mathbb{R}} x b y$.

The non-degenerate case is too long to reproduce here in full, but a sketch may be interesting; the full formalized proof can be found at [14, pages 32-34].

Recall that the non-degenerate case occurs when $X \nsubseteq\{a\}$ and $Y$ is non-empty. First, a point $c \in X-\{a\}$ is chosen. As will be explained, the segment $a c$ is effectively chosen as a unit to measure the positions of other points along the line, with $a$ acting as the origin; Figure 5.5 may assist in understanding this.

For each $y \in Y$, we have $\mathrm{B} a c y$, which, by the definition of betweenness in this model, allows us to conclude that there is a scalar $j$ such that $y-a=j(c-a)$; furthermore, $j \geq 1$. 


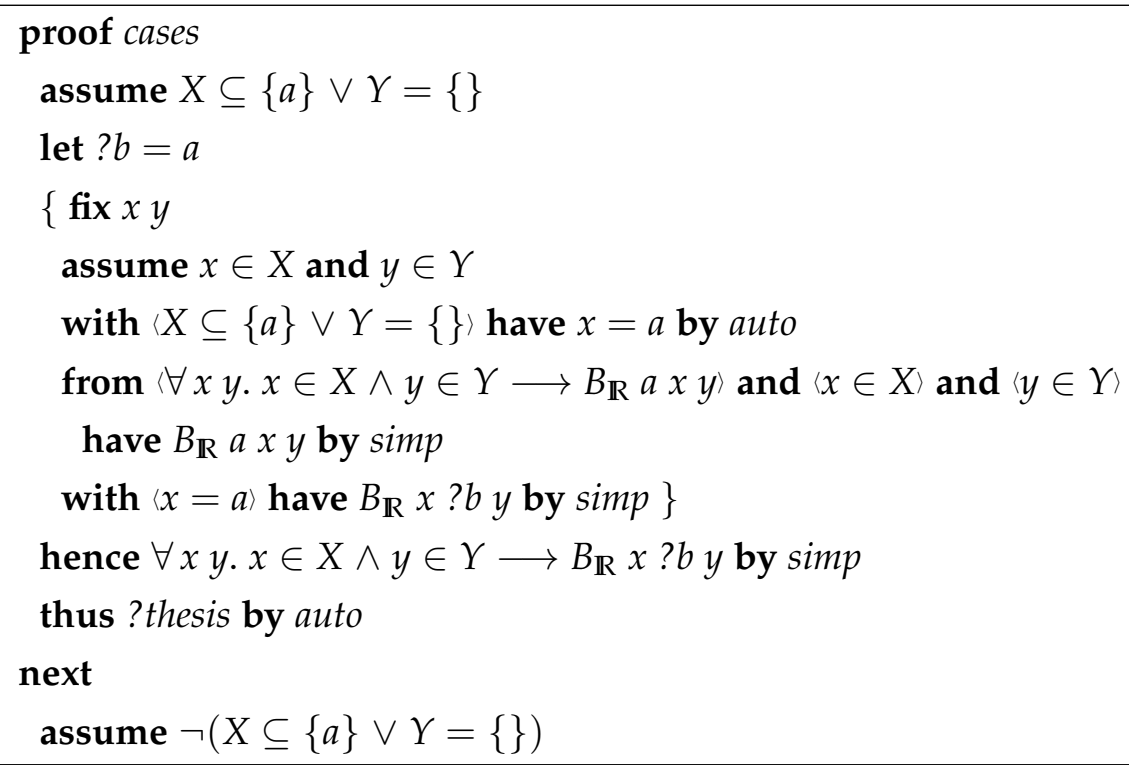

Figure 5.4: Degenerate cases considered simultaneously

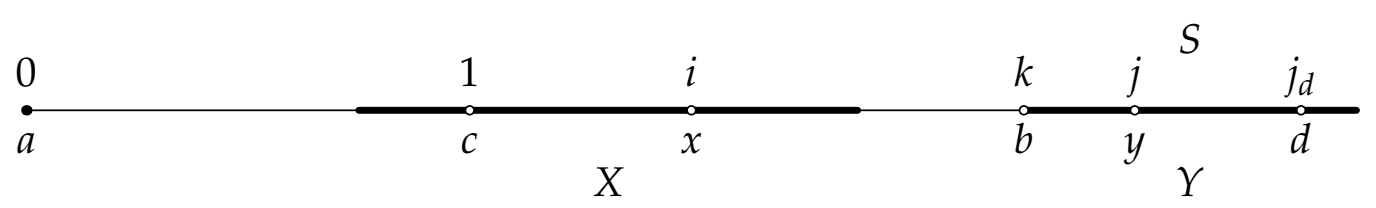

Figure 5.5: Measuring distances from $a$ using the segment $a c$ 
Because $Y$ is non-empty, we can choose a point $d \in Y$. Associated with $d$ is the scalar $j_{d}$ such that $d-a=j_{d}(c-a)$.

For each $x \in X$, we have $\mathrm{B}$ axd, which allows us, via $j_{d}$, to prove the existence of a scalar $i$ such that $x-a=i(c-a)$; thus each element of $X$ or $Y$ is equal to $a$ plus some scalar multiple of $c-a$.

Now consider the set $S$ of scalars $j$ such that $a+j(c-a) \in Y$. It is nonempty, because $j_{d} \in S$, and it is bounded below, by 1 ; therefore $S$ must have an infimum, say $k$. The point $b$ is chosen so that $b-a=k(c-a)$; essentially, $b$ can be thought of as the "right-most left-hand bound" of the set $Y$, if the picture is oriented as in Figure 5.5 (although other choices of $b$ may have been possible, as in Figure 2.6).

Given an arbitrary $x \in X$, choose $i$ such that $x-a=i(c-a)$. Because $\mathrm{B}$ axy for each $y \in Y$, it can be shown that $i$ is a lower bound of $S$; because $k$ is the greatest lower bound, we have $i \leq k$. For arbitrary $y \in Y$, we can choose $j$ such that $y-a=j(c-a)$; then $j \in S$, and since $k$ is a lower bound of $S$, we have $k \leq j$. Finally, with $i \leq k \leq j$, we can show that $\mathrm{B} x b y$ always holds, as required.

\subsection{The Euclidean axiom}

In Section 2.4, after stating Tarski's version of the Euclidean axiom, we informally considered the way in which the axiom succeeds in Euclidean geometry; the proof that $\mathbb{R}^{n}$ satisfies the Euclidean axiom is similar in spirit to the explanation given there. However, we can now refer specifically to coordinates in $\mathbb{R}^{n}$, rather than points in some generic model of Euclidean geometry; as a result, we can write a proof that avoids treating as a degenerate case the situation where $a, b$, and $d$ are collinear.

Suppose we are given $a, b, c, d$, and $t$ that satisfy the hypotheses of the axiom. Because $\mathrm{B} a d t$, there is some $j$ such that $d-a=j(t-a)$; because $a \neq d$, we have $j \neq 0$. We can then choose $x$ and $y$ such that $b-a=j(x-a)$ and $c-a=j(y-a)$ (see Figure 5.6). These choices of $x$ 


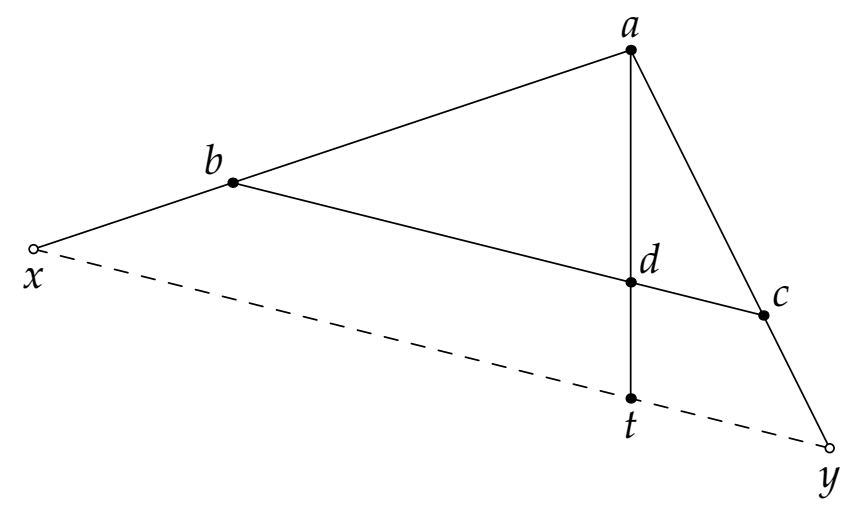

Figure 5.6: A construction for Tarski's Euclidean axiom

and $y$ immediately ensure that $\mathrm{B} a b x$ and $\mathrm{B} a c y$; using the fact that $\mathrm{B} b d c$, a little rearrangement reveals that it is also the case that $\mathrm{B} x t y$, as required.

The above proof is an informal summary of the proof that was formalized in Isabelle - see [14, page 35].

Another way to understand this construction is as follows: the figure $a b c d$ is dilated (with centre $a$ ) so that the image of $d$ is $t$; the images of $b$ and $c$ are chosen to be the points $x$ and $y$, respectively. 


\section{Chapter 6}

\section{The independence of the Euclidean axiom}

\subsection{Existing proofs}

As explained in Section 1.3, the independence of the parallels postulate can be immediately derived from Beltrami's work published in 1868 . However, this "independence" result is imprecise unless we specify a particular set of axioms from which a particular version of the parallels postulate is claimed to be independent. It is meaningful to say that Beltrami's work establishes the independence of Euclid's parallels postulate from Euclid's other axioms; however, as discussed in Section 2.1, Euclid's axioms are not suitable for highly formalized mechanical reasoning.

We may reasonably ask who first proved the independence of Tarski's Euclidean axiom from Tarski's other axioms of plane geometry, or where such a proof is published. Such a proof seems very difficult to find.

In [21, page 208], given an ordered field $\mathfrak{F}$, a structure $\mathfrak{K}_{n}(\mathfrak{F})$ is defined that is intended to be a model of an $n$-dimensional hyperbolic version $\mathcal{H}_{n}$ of Tarski's axioms of geometry. It is then asserted that for a natural number $n \geq 2$, a given structure is a model of $\mathcal{H}_{n}$ if and only if it is 
isomorphic to $\mathfrak{K}_{n}(\mathfrak{F})$ for some Euclidean* ordered field $\mathfrak{F}$. As well as leading up to a categoricity result on the same page, this assertion entails the independence of Tarski's Euclidean axiom from his other ten axioms of plane geometry.

The proof of the assertion is omitted from [21], but several citations are given for the various results asserted on that page, without any indication about which citations contain the proofs of which assertions. I believe that the only citation relating to the assertion that the structure is a model of the axioms is Szmielew's [26]. Indeed, [18, page 333] also repeats a version of the same assertion, and cites [26] for the proof (with a note to see also [21]).

Unfortunately, where Szmielew states the theorem in question (see [26, page 49]), her proof begins by asserting that her model is "well known" to be a model of the axioms in question; she proves in more detail the other part of the theorem - that every model of the hyperbolic axioms is isomorphic to her model over some field. The assertion that the result we are interested in is "well known" is slightly curious, because the paper immediately preceding [26] is [28], which may have been only the second time that any version of Tarski's axioms was published, the first having been in an endnote in [27, pages 55-57] (see [29, pages 188-189]).

As will be seen in the rest of this thesis, this "well known" independence result is not necessarily trivial to prove formally, although it may still be reasonable to assert that it was well known in an informal sense, on the basis of what was known about various models of hyperbolic geometry, and about the theorems that hold in those models.

The difficulty of the proof will, of course, depend on the model chosen, and different models might make different axioms easier or more difficult to verify; furthermore, formalization of the independence result may be aided by existing published proofs that particular models satisfy

${ }^{*}$ A field $\mathfrak{F}$ is called Euclidean if for each $a \in \mathfrak{F}$, either $a$ or $-a$ is the square of an element of $\mathfrak{F}$; see [21, page 225]. 
particular axioms. In Section 6.2 we discuss the choice of a hyperbolic model for our formal verification.

\subsection{Choosing the model}

Considering only the 2-dimensional real case, the model given in [21, page 208] uses the open unit disc in $\mathbb{R}^{2}$ as its set of points; betweenness is defined as in the Cartesian model of Euclidean geometry (see Section 5.1 ); congruence is defined so that $x y \equiv u v$ is true if and only if

$$
\frac{(1-x \cdot y)^{2}}{\left(1-\|x\|^{2}\right)\left(1-\|y\|^{2}\right)}=\frac{(1-u \cdot v)^{2}}{\left(1-\|u\|^{2}\right)\left(1-\|v\|^{2}\right)}
$$

Some features of this model make it a good candidate to work with for formal verification. For example, because betweenness is defined as in the Cartesian model, some of the results about betweenness in the Cartesian plane may be used to verify that axioms about betweenness also hold in this model of the hyperbolic plane. Also, the definition of congruence suggests a semimetric distance function where the distance between $x$ and $y$ is defined to be

$$
\frac{(1-x \cdot y)^{2}}{\left(1-\|x\|^{2}\right)\left(1-\|y\|^{2}\right)}-1
$$

Verifying that this does indeed define a semimetric space would allow us to immediately conclude that the definition of congruence satisfies Tarski's first three axioms.

I know of no published proof that a model with this definition of congruence satisfies, for example, the five-segments axiom (or any of the other relatively difficult axioms).

Other candidate models of the hyperbolic plane include the so-called Poincaré disc and Poincaré half-plane models (both of which are originally due to Beltrami - see [24, pages 263-266]). In these models, most lines are represented by arcs of circles in the Cartesian plane, complicating 
the definition of betweenness. Because betweenness is one of Tarski's primitive relations, a simple definition is preferable.

Another model is defined by Borsuk and Szmielew in [3, pages 245250], and is called the Klein-Beltrami model. It is very similar to the model in [21, page 208], but it is defined in the projective plane, rather than in $\mathbb{R}^{2}$.

A bijection is fixed between the projective plane (minus a single line) and $\mathbb{R}^{2}$. The open unit disc in $\mathbb{R}^{2}$ (or rather, its image in the projective plane) is again used as the set of points in the model of the hyperbolic plane; the circle bounding the disc is called the absolute.

Again, betweenness is defined to be equivalent to betweenness in the Cartesian plane, so that results about betweenness in the Cartesian plane can be used to establish similar results about betweenness in the KleinBeltrami model of the hyperbolic plane.

If an invertible linear transformation from the projective plane to itself (what is sometimes called a collineation - see [3, page 233]) maps the absolute to itself, then it is called a K-isometry. Congruence in the KleinBeltrami model is then defined so that $a b \equiv c d$ is true if and only if there is a $K$-isometry $f$ such that $f(a)=c$ and $f(b)=d$.

While retaining the advantage of a simple definition of betweenness, this model has the additional advantage that it is built in the projective plane, where any two lines must intersect; this means that proofs need not consider degenerate cases where two lines that usually intersect may sometimes be parallel. Furthermore, Borsuk and Szmielew published proofs verifying that the Klein-Beltrami model satisfies their own axioms of geometry (see [3, pages 250-258]); some of their axioms are very similar to some of Tarski's axioms, so the published proofs could be considered likely to assist in writing the necessary computer-verifiable proofs for this project.

These advantages were sufficient to encourage me to choose Borsuk and Szmielew's Klein-Beltrami model for the computer verification of the 
independence of Tarski's Euclidean axiom. As will be seen in Chapter 8, the advantage of access to Borsuk and Szmielew's published proofs was not always as great as was anticipated, either because their justification was insufficient, or because following their proof would have required formalizing too many extra concepts and theorems about them. Nevertheless, Borsuk and Szmielew's [3] was quite helpful during this project, and I referred to it frequently during my formalization work. 


\section{Chapter 7}

\section{Formalizing the projective plane}

\subsection{Overview}

In order to formalize the Klein-Beltrami model of the hyperbolic plane, it was first necessary to define the projective plane, in which the KleinBeltrami model is defined. Of course, a definition on its own is not particularly useful; theorems about the projective plane must also be formally proven. This proved to be a larger task than initially expected. One file was used to collect the formalized theorems about the projective plane and about a bijection between (most of) it and the Cartesian plane; this file is over three thousand lines long - more than half the length of the file used to formalize the Klein-Beltrami model itself and prove the necessary theorems about it.

Section 7.2 describes the formalization of the points of the projective plane. Then, Section 7.3 uses the points of the projective plane as an example to explain how much detail is required in formal proofs when repeatedly switching between an abstract concept and its representation. Section 7.4 covers the way in which projective lines were formalized in Isabelle, and Section 7.5 covers collineations of the projective plane. Finally, Section 7.6 explains a particular bijection between the Cartesian plane and most of the projective plane, and how it was formalized in Isabelle. 


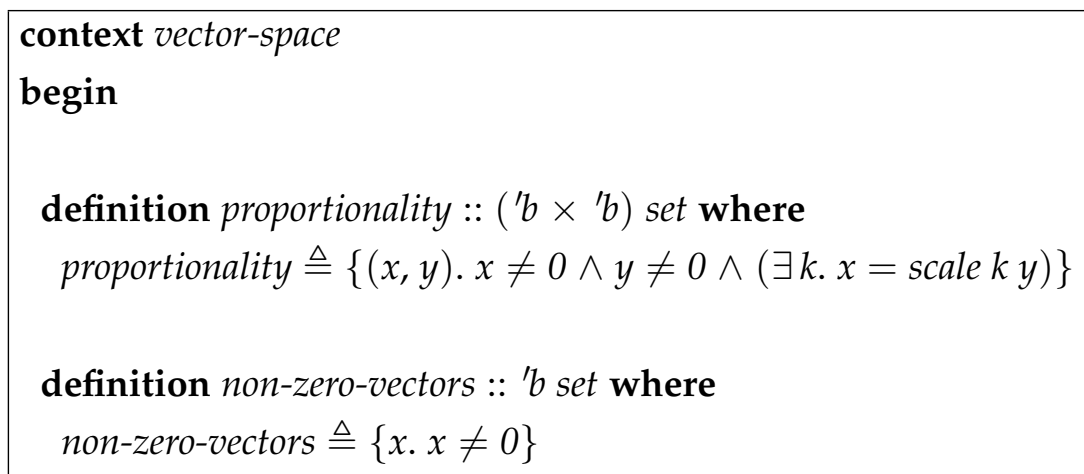

Figure 7.1: Definition of proportionality and non-zero vectors

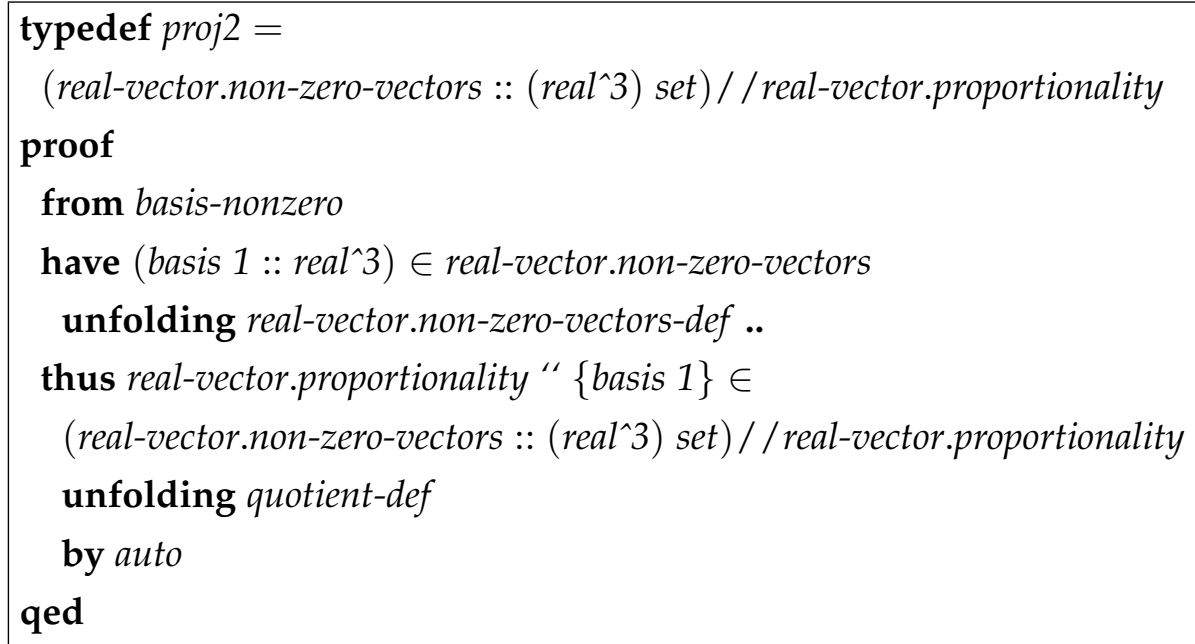

Figure 7.2: Defining a type for the points of the real projective plane

\subsection{Points of the projective plane}

The first step in formalizing the projective plane was to formalize proportionality, which is an equivalence relation on non-zero vectors. See Figure 7.1 for the definition, which is made in the context of the locale called vector-space; the proof that it is an equivalence relation on non-zero vectors can be found at [14, pages 53-54].

Next, a new type is defined to represent points of the real projective plane - see Figure 7.2. 
Isabelle allows the definition of a new type that is isomorphic to a nonempty subset of an existing type. In this case, the new type is isomorphic to the partition of non-zero real vectors of dimension three that is defined by the equivalence relation of proportionality.

Because Isabelle does not allow empty types, the user must supply a proof (if one cannot be found automatically) that the subset defining the new type is non-empty. In Figure 7.2, basis 1 is a particular element of the standard basis of $\mathbb{R}^{3}$, and real-vector.proportionality " $\{$ basis 1$\}$ is the cell in which basis 1 belongs in the partition defined by proportionality.

\subsection{Abstraction and representation}

After the new type proj2 is successfully defined, Isabelle automatically defines representation and abstraction functions, Rep-proj2 and Abs-proj2, respectively. The former is from the type proj2 to the type (real^3) set, and the latter from (real^3) set to proj2.

Given a point $p$ of type proj2, we can write Rep-proj2 $p$, which is the cell of the partition that represents the point $p$.

Conversely, given a subset $S$ of $\mathbb{R}^{3}$, we can write Abs-proj2 $S$. If $S$ is a cell of the partition, then Abs-proj2 $S$ is the point of type proj2 that the cell represents; if $S$ is some subset of $\mathbb{R}^{3}$ that is not a cell of the partition, then Abs-proj2 $S$ is an arbitrary point of proj 2 - soundness is guaranteed because we were forced to prove that this type is non-empty.

However, instead of working with cells of a partition, mathematicians often choose to work with representative members of the cells instead; it would be more convenient to have representation and abstraction functions directly between the types proj2 and real^3. These functions were defined as in Figure 7.3. The symbol $\epsilon$ in the definition of proj2-rep represents Hilbert's indefinite description operator; an arbitrary element of the appropriate cell is chosen to be the real-vector representative of a given point in proj2. 


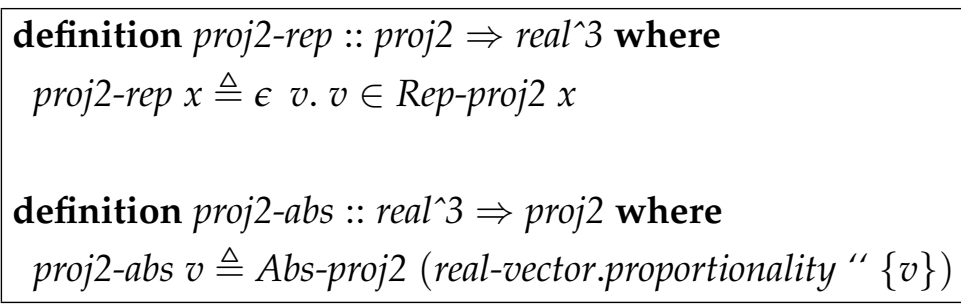

Figure 7.3: Useful abstraction and representation functions for proj2

In ordinary mathematical prose, mathematicians often silently employ representation and abstraction functions without introducing or consistently using notation for them; they leave their readers to convince themselves that everything can be made rigorous if necessary. Of course, this is not possible with computer verifiable proofs; the computer demands that every detail is made rigorous, either by automatic proof search or by the human author of the proof.

To illustrate the level of detail that explicit abstraction and representation require, see Figure 7.4, the proof that taking the representative of a point and abstracting again behaves as the identity function. This is only one of many similar technical lemmas; consider the proof that if $v$ is non-zero, then proj2-rep (proj2-abs $v$ ) is a non-zero scalar multiple of $v$, the proof that for non-zero vectors $v$ and $w$, we have proj2-abs $v=$ proj2-abs $w$ if and only if $v$ and $w$ are scalar multiples of each other, and so on. Then, when we later formalize lines in and collineations of the projective plane, we must prove many similar theorems for each of those types, as well as theorems about the interactions between the types.

For this reason, proj2-abs and proj2-rep appear frequently in this formalization, highlighting by contrast how often mathematical prose glosses over abstraction and representation. This is both encouraging and worrying: encouraging because it demonstrates mathematicians' ability to quickly and accurately convince themselves that the necessary abstraction and representation steps can be rigorously introduced; worrying because the mathematicians in question are not always perfectly accurate, 


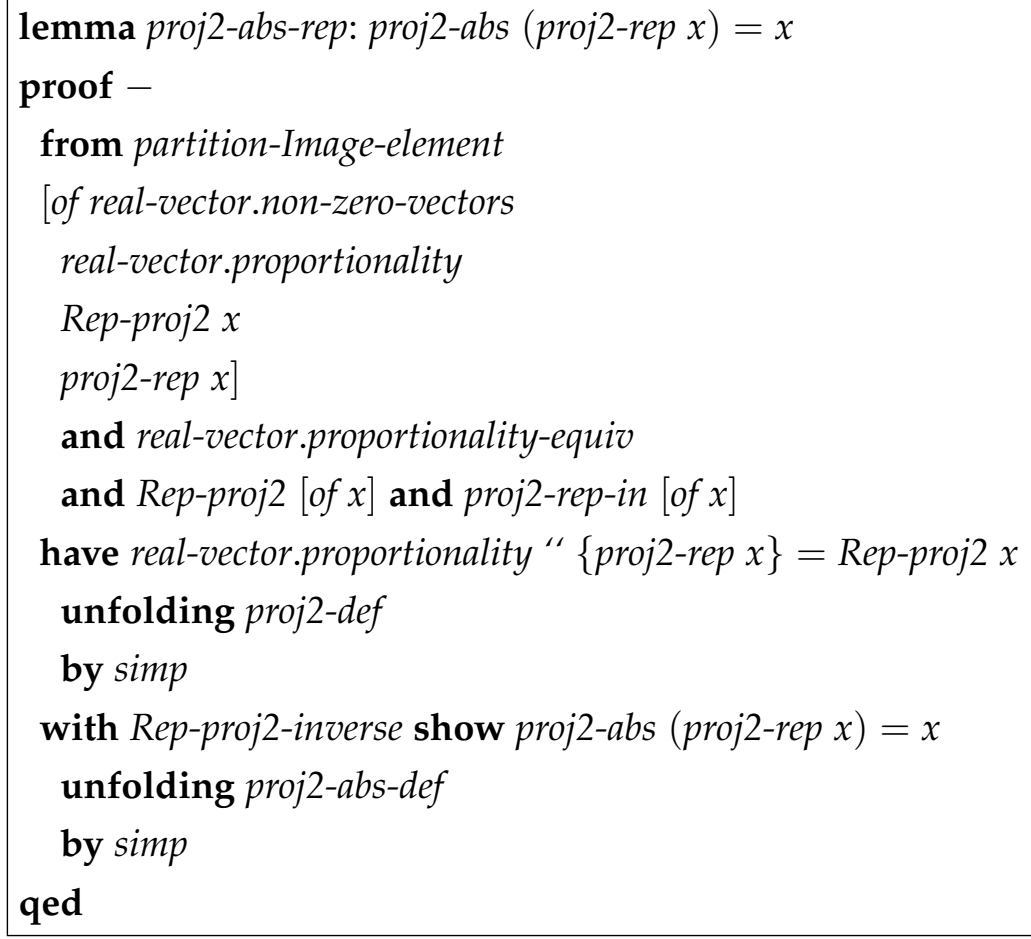

Figure 7.4: Abstracting a representative 
and sometimes a fully rigorous proof is not as straightforward as the published text suggests.

The frequent use of abstraction and representation functions in this project might have been mitigated if Isabelle's quotient types had been available when the project was started.

\subsection{Lines in the projective plane}

Collinearity in the projective plane can be characterized in several equivalent ways.

The points of the projective plane are represented by cells of a partition of $\mathbb{R}^{3}-\{(0,0,0)\}$. The partition is defined by the equivalence relation of proportionality, so that each cell is almost a line through the origin in $\mathbb{R}^{3}$; by inserting $(0,0,0)$ into each cell, we can define a bijection between points of the projective plane and lines through the origin in $\mathbb{R}^{3}$.

Similarly, lines of the projective plane can be represented by planes through the origin in $\mathbb{R}^{3}$; a point $p$ of the projective plane is incident with a line $l$ if and only if the line in $\mathbb{R}^{3}$ representing $p$ is a subset of the plane representing $l$.

However, just as it is convenient to represent points of the projective plane by individual vectors in $\mathbb{R}^{3}$ instead of cells of a partition, it is also convenient to represent lines of the projective plane in a similar manner. It may seem natural to represent lines by pairs of vectors in $\mathbb{R}^{3}$ whose span is the representative plane; however, in the case of the projective plane - as opposed to higher-dimensional projective spaces - it is possible to represent lines even more simply: by non-zero vectors of $\mathbb{R}^{3}$ that are normal to the representative plane.

This representation has the benefit that if $u$ is a vector representing a point of the projective plane, and $v$ a vector representing a line, then the point and line are incident if and only if $u \cdot v=0$. This was the manner in which lines in the projective plane were formalized in Isabelle, although 
datatype proj2-line $=P 2 L$ proj2

definition $L 2 P$ :: proj2-line $\Rightarrow$ proj2 where

$L 2 P l \triangleq$ case $l$ of $P 2 L p \Rightarrow p$

Figure 7.5: Defining a type for lines of the projective plane

definition proj2-line-abs :: real^3 $\Rightarrow$ proj2-line where

proj2-line-abs $v \triangleq P 2 L$ (proj2-abs $v)$

definition proj2-line-rep :: proj2-line $\Rightarrow$ real^3 where

proj2-line-rep $l \triangleq$ proj2-rep (L2Pl)

Figure 7.6: Abstraction and representation functions for proj2-line

collinearity of three points was also characterized in an equivalent way: three points are collinear if and only if their representative vectors in $\mathbb{R}^{3}$ are linearly dependent (see [14, pages 59-73]).

Instead of formalizing lines of the projective plane by repeating the entire process required to formalize points, it was more convenient to formalize the type proj2-line of lines as a new type isomorphic to the type of points, with trivial abstraction and representation functions between them - P2L and L2P. Figure 7.5 shows the definition. Of course, the purpose was to represent lines of the projective plane by vectors in $\mathbb{R}^{3}$, so representation and abstraction functions were defined for that purpose, too - see Figure 7.6.

With these definitions, it was possible to easily lift proofs about points of the projective plane to prove similar results about lines; for example, compare Figure 7.7 with Figure 7.4. 
lemma proj2-line-abs-rep [simp]: proj2-line-abs (proj2-line-rep $l)=l$

unfolding proj2-line-abs-def and proj2-line-rep-def

by (simp add: proj2-abs-rep)

Figure 7.7: Abstracting a representative using a similar theorem

\subsection{Collineations of the projective plane}

As was mentioned in Section 6.2, a collineation of the projective plane is an invertible linear transformation from the projective plane to itself. A collineation can be represented by a $3 \times 3$ invertible matrix. If $u$ is a non-zero (row) vector and $C$ is an invertible matrix, then the collineation represented by $C$ maps the point represented by $u$ to the point represented by $u C$.

For any non-zero scalar $k$, the point represented by $u C$ is equal to the point represented by $u(k C)$, so the matrix $k C$ represents the same collineation as $C$; apart from scalar multiples of $C$, no other matrices represent the same collineation as $C$. Therefore, the equivalence classes of invertible $3 \times 3$ matrices under proportionality were used in Isabelle to define a type $\operatorname{cltn} 2$ of collineations of the projective plane. As with the points and lines of the projective plane, useful abstraction and representation functions were defined - this time between $\operatorname{cltn} 2$ and real $3^{\wedge} 3$, the type of $3 \times 3$ matrices with real entries. For these definitions, see [14, page 77].

Collineations can be composed and inverted, and the collineation represented by the identity matrix acts as the identity collineation. In this way, the collineations form a group; this was formalized in Isabelle - see [14, pages 81-84].

A function was defined to apply a collineation to a point of the projective plane - see Figure 7.8.

Collineations can also be applied to lines of the projective plane, but care needs to be taken. If $v$ is the (column) vector representing a line, and 
definition apply-cltn2 :: proj2 $\Rightarrow$ cltn2 $\Rightarrow$ proj2 where

apply-cltn2 $x A \triangleq$ proj2-abs (proj2-rep $x v *$ cltn2-rep $A$ )

Figure 7.8: Applying a collineation to a point

definition apply-cltn2-line :: proj2-line $\Rightarrow$ cltn2 $\Rightarrow$ proj2-line where

apply-cltn2-line $l A$

$\triangleq P 2 L($ apply-cltn2 (L2Pl) (cltn2-transpose (cltn2-inverse A)))

Figure 7.9: Applying a collineation to a line

$C$ is a matrix representing a collineation, then $C^{-1} v$ represents the image of the line under the collineation.

To understand this, recall from Section 7.4 that if $u$ and $v$ represent a point and a line, respectively, then $u \cdot v=0$ if and only if the line and the point are incident with each other; now that we consider $u$ to be a row vector and $v$ to be a column vector, $u v=(0)$ characterizes incidence.

So, the images of the point and line are incident with each other if and only if $(u C)\left(C^{-1} v\right)=(0)$, which is equivalent to $u v=(0)$. Therefore, the collineation preserves incidence, proving that it deserves the name "collineation".

The formalization of the application of collineations to projective lines is shown in Figure 7.9. The Isabelle functions $P 2 L$ and $L 2 P$ can be thought of as transposing the representative vectors, to maintain the idea that points are represented by row vectors and lines by column vectors.

Once these functions (apply-cltn2 and apply-cltn2-line) have been defined, it can then be shown that they each define a group action (see [14, pages 84-87]). In order to formalize this, it was first necessary to write a brief formalization of group actions (see [14, pages 51-52]), but groups were already formalized. 
definition cart2-pt :: proj2 $\Rightarrow$ real`2 where

cart2-pt $p \triangleq$

vector [(proj2-rep $p) \$ 1 /($ proj2-rep $p) \$ 3,($ proj2-rep $p) \$ 2$ / (proj2-rep $p) \$ 3]$

Figure 7.10: A map from the projective plane to the Cartesian plane

\subsection{A mapping to the Cartesian plane}

As was mentioned in Section 6.2, Borsuk and Szmielew's Klein-Beltrami model of the hyperbolic plane relies on fixing a bijection between the Cartesian plane and most of the projective plane. Many suitable bijections are possible; in fact, any line of the projective plane can be deleted, and the remainder of the projective plane can be put in bijection with the Cartesian plane in a way that preserves collinearity.

For our purposes, we must choose a specific bijection. The bijection used in [3] and in this project is as follows. The Cartesian point $(x, y)$ is mapped to the projective point represented by $(x, y, 1)$; for the inverse, a projective point represented by $(x, y, z)$ (with $z \neq 0$ ) is mapped to the Cartesian point $\left(\frac{x}{z}, \frac{y}{z}\right)$; the line where $z=0$ (that is, the line represented by $\left.(0,0,1)^{\top}\right)$ is the line deleted from the projective plane for the purposes of this bijection.

When this was formalized in Isabelle (see [14, pages 106-109]), the map from real`2 to proj2 was called proj2-pt, and was untroublesome. The inverse map was defined from all of proj2 (without deleting a line) to

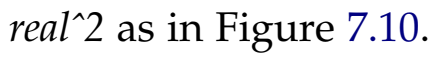

This may appear to be ill-defined when the third coordinate of the representative is 0 , but Isabelle makes division a total function by specifying that $\frac{x}{0}=0$. Theorems about division must then specify that the denominator is non-zero (unless the theorem also happens to hold with Isabelle's definition of division). For example, the statement of one of the theorems expressing that proj2-pt and cart2-pt are inverses is shown in Figure 7.11; it is necessary to include the hypothesis that the third co- 
lemma proj2-cart2:

assumes $z$-non-zero $p$

shows proj2-pt (cart2-pt $p)=p$

Figure 7.11: proj2-pt and cart2-pt are inverses

ordinate of the representative of $p$ is non-zero (for which an abbreviation has been previously defined - see [14, pages 106-107]).

For this project, it is important that this bijection preserves collinearity; that is, our characterizations of collinearity in the projective plane must coincide with collinearity in the Cartesian plane when this bijection is applied. Several theorems expressing this in different ways were formalized in Isabelle; these can be found at [14, pages 109-115]. 


\section{Chapter 8}

\section{Formalizing our model of the hyperbolic plane}

\subsection{Defining the model}

As mentioned in Section 6.2, the set of points of the hyperbolic plane is represented by the open unit disc in $\mathbb{R}^{2}$, or by its image in the projective plane, according to our fixed bijection. Specifically, we consider in the Cartesian plane the unit circle centred at $(0,0)$; its image in the projective plane is taken as the absolute, and the image of its interior is taken as the set of projective points representing the points of the hyperbolic plane; the former set we call $S$ and the latter $K_{2}$, following [3, page 245].

This situation can be characterized more directly in the projective plane by fixing a matrix

$$
M=\left(\begin{array}{ccc}
1 & 0 & 0 \\
0 & 1 & 0 \\
0 & 0 & -1
\end{array}\right)
$$

The points of the absolute $S$ are the projective points whose representatives $u$ satisfy $u M u^{\top}=(0)$, and the points of $K_{2}$ (representing the points of the hyperbolic plane) are the projective points whose representatives 
definition real-hyp2-B :: [hyp2, hyp2, hyp2] $\Rightarrow$ bool

$\left(B_{K}\right.$ - - $\left.[99,99,99] 50\right)$ where

$B_{K} p q r \triangleq B_{\mathbb{R}}($ hyp2-rep $p)($ hyp2-rep $q)($ hyp2-rep $r)$

Figure 8.1: Lifting betweenness from the Cartesian plane

in $\mathbb{R}^{3}$ satisfy $u M u^{\top}=(x)$, with $x<0$.

Furthermore, given a projective point $p$ represented by a vector $u$, the polar of $p$ with respect to the conic is represented by $M u^{\top}$; similarly, if $v$ represents a projective line $l$, then the pole of $l$ with respect to the conic is represented by $v^{\top} M^{-1}=v^{\top} M$ (because our choice of $M$ has $M^{-1}=M$ ).

In fact, any conic in the projective plane can be characterized in this way by replacing $M$ with a different symmetric matrix (see [32, page 177]). This project needs only this specific conic, characterized using this specific matrix $M$; see [14, pages 119-124] for the formalization.

A new type hyp2 is defined to represent the points of the hyperbolic plane; application of collineations is lifted to this new type, and $K$-isometries are defined to be collineations that map the absolute to itself; these are easily shown to form a subgroup of collineations. Finally, as promised in Section 6.2, congruence of the hyperbolic plane is defined so that $a b \equiv c d$ if and only if there is a K-isometry $f$ such that $f(a)=c$ and $f(b)=d$. See $[14$, pages $133,136-138]$ for the formalized definitions and results mentioned in this paragraph.

Betweenness of the hyperbolic plane is lifted from betweenness in the Cartesian plane; see Figure 8.1. In that figure, hyp2-rep has already been defined so that hyp2-rep $p$ denotes the Cartesian equivalent of the projective representative of the hyperbolic point $p$.

\subsubsection{Preliminary results}

An important theorem about $K$-isometries is that if $f$ is a $K$-isometry and $p \in K_{2}$, then $f(p) \in K_{2}$. As suggested in [3, pages 245-246], this can 
be proven by first characterizing points in $K_{2}$ as points $p$ such that every line through $p$ intersects $S$ twice; because collineations are invertible and preserve incidence of points with lines, and because $K$-isometries in particular preserve incidence of points with the absolute, the result follows; see [14, pages 138-152] for the formalization.

This result can then be used to establish that the application of $\mathrm{K}$ isometries defines a group action on points of the hyperbolic plane - see [14, pages 154-155].

In order to formalize the theorem mentioned above that all $\mathrm{K}$-isometries map $K_{2}$ to itself, it was first necessary to formalize the quadratic formula in Isabelle (see [14, pages 115-118]). The omission of this from Isabelle is surprising, given how much non-trivial mathematics is available in Isabelle, including, for example, the Hahn-Banach theorem. It is especially surprising considering that the general solutions of cubics and quartics are said to have been formalized in Isabelle (see [34]), although I was unable to find the proofs of these either in the Isabelle release or in Isabelle's Archive of Formal Proofs [13]; I also sent a query to the Isabelle users' email list asking about existing work on discriminants of quadratics, but I received no response.

\subsection{The reflexivity axiom for equidistance}

It took a surprisingly long time to formalize the proof that the KleinBeltrami model of the hyperbolic plane satisfies the first of Tarski's axioms - the reflexivity axiom for equidistance. It would have been trivial to verify it if the definition of congruence had been changed so that $a b \equiv c d$ is defined to be true if and only if there is a $K$-isometry $f$ such that $f(a)=d$ and $f(b)=c$; the identity $K$-isometry would have sufficed.

Although this definition of congruence would have been equivalent to the one chosen, it would merely have postponed the difficulties encountered until the verification of the second axiom - the transitivity axiom 
for equidistance. With the definition that was used, the second axiom is a consequence of the fact that the application of $K$-isometries defines a group action on the points of the hyperbolic plane. (The third axiom is also a consequence of this fact, whichever definition of congruence is used.)

Borsuk and Szmielew's Statement 68 (see [3, page 249]) is the essence of what must be proven in order to show that Tarski's first axiom holds in the Klein-Beltrami model of the hyperbolic plane: given $a$ and $b$ in $K_{2}$, there is a K-isometry $f$ such that $f(a)=b$ and $f(b)=a$. The proof formalized in Isabelle follows the general outline of Borsuk and Szmielew's proof. This required first formalizing some theorems about the projective plane, such as part of Borsuk and Szmielew's Statement 53 (see [3, page 240]): essentially, in the projective plane, any four points in general position can be mapped to any other four points in general position by some collineation; see [14, pages 90-96] for the formalization.

\subsubsection{Abstraction and representation in the proof of State- ment 66}

During the attempt to formalize Borsuk and Szmielew's proof of Statement 68 , one clear example arose of the tendency of ordinary mathematical prose to take abstractions and representations for granted. This arose in the formalization of the proof of part of Borsuk and Szmielew's Statement 66 (see [3, pages 247-248]). For our purposes, the pertinent part of Statement 66 says that given $a_{1}, a_{2} \in K_{2}$ and $p_{1}, p_{2} \in S$, there is a $K$-isometry $f$ such that $f\left(a_{1}\right)=a_{2}$ and $f\left(p_{1}\right)=p_{2}$.

Borsuk and Szmielew's proof of Statement 66 involves some constructions shown in Figure 8.2. Given $a \in K_{2}$ and $p \in S$, first construct the other intersection of line $a p$ with the absolute $S$; this is possible because any line through a point in $K_{2}$ (such as a) must intersect $S$ twice.

Next, construct the tangents to $S$ at $p$ and $q$. (Note that the tangent at 


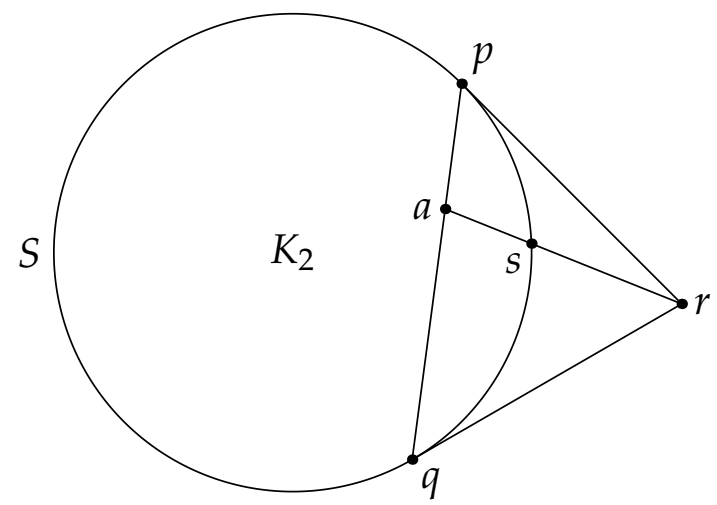

Figure 8.2: Constructions for the proof of Statement 66

a point of $S$ is the polar of that point.) Borsuk and Szmielew then take $r$ to be the intersection of these two tangents. They do not consider the degenerate case that would occur in the Cartesian plane when $p$ and $q$ are at diametrically opposite points; in this case, the tangents would not intersect, so Borsuk and Szmielew must be making this construction not in the Cartesian plane, but in the projective plane, where any two lines intersect.

On the other hand, in order to construct s, Borsuk and Szmielew then appeal to their "Theorem 37", by which they mean "Statement 37" (see [3, page 229]); this statement is about the Cartesian plane, and they have not explicitly shown how it can be lifted to the projective plane.

Instead of formalizing Statement 37 in the Cartesian plane and then lifting it to the projective plane, it was simpler to formally prove the necessary result directly; indeed, it is an immediate consequence of the fact (already discussed) that any line through a point in $K_{2}$ must intersect $S$ twice, although only one of the intersections was needed in this case.

Borsuk and Szmielew chose to use their Statement 37 because their Statement 66 requires $s$ to be in a particular arc of $S$; the Isabelle formalization proves only statement66-existence (see [14, pages 160-165]), which is weaker, and which is not sensitive to which intersection of line ar with 
$S$ is chosen as the point $s$.

Borsuk and Szmielew's casual appeal to a Cartesian theorem in a projective construction serves to illustrate the way in which mathematical prose often glosses over questions of abstraction and representation. This can make proofs more difficult to formalize than the prose suggests; if the Isabelle formalization had proven all of Statement 66, and had followed Borsuk and Szmielew's proof, it would have been much more complicated than it is - not least because arcs of $S$ would first need to have been formalized, lifting them from arcs in the Cartesian plane.

\subsubsection{Proof that Statement 66 and axiom 1 hold}

The rest of the proof of the relevant part of Statement 66 proceeds as follows. The constructions of Figure 8.2 are performed for $a_{1}$ and $p_{1}$, and also for $a_{2}$ and $p_{2}$. Then, where $i=1$ or $i=2$, it is the case that $p_{i}, q_{i}, r_{i}$, and $s_{i}$ are in general position (see [14, pages 155-157]), so a collineation $f$ is chosen such that $f\left(x_{1}\right)=x_{2}$, for $x \in\{p, q, r, s\}$. Using a version of Borsuk and Szmielew's Statement 65 (see [3, page 247] and [14, pages 157-160]), we can ensure that $f$ is a $K$-isometry. We already have $f\left(p_{1}\right)=p_{2}$, and because collineations preserve incidence of points with lines, and $a_{i}$ is the intersection of lines $p_{i} q_{i}$ and $r_{i} s_{i}$, we can also conclude that $f\left(a_{1}\right)=a_{2}$, as required.

Finally, in order to prove that Tarski's axiom 1 holds, we must show that given $a, b \in K_{2}$, there is a K-isometry $f$ such that $f(a)=b$ and $f(b)=a$. If $a=b$, the identity $K$-isometry suffices as a choice for $f$; otherwise, let $p$ and $q$ denote the two intersections of line $a b$ with $S-$ see Figure 8.3. By Statement 66 , choose a K-isometry $f$ such that $f(a)=b$ and $f(p)=q$.

Because $f$ is a collineation, it preserves incidence of points with lines, so it is easily seen that it maps the line $a b$ to itself. Because $f$ is a $K$ isometry, it also preserves incidence of points with $S$. Therefore, $f(q)$ 


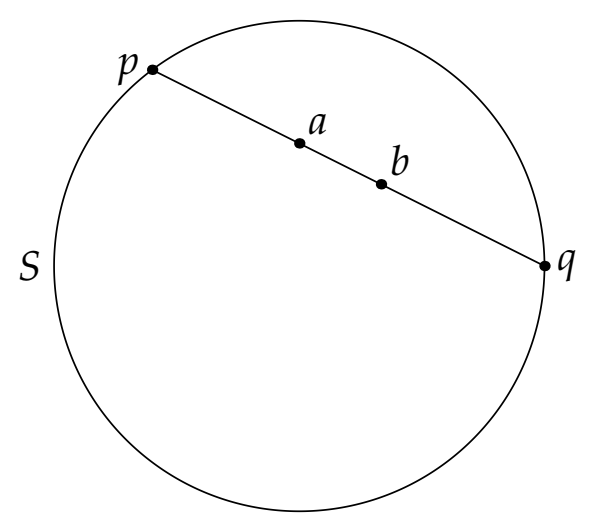

Figure 8.3: Constructions for the verification of axiom 1

must be one of the two intersections of line $a b$ with $S$. We cannot have $f(q)=q$, because $f(p)=q$ and $f$ is invertible, but $p \neq q$. Therefore, the only possibility is that $f(q)=p$.

Because $f$ is a collineation such that $f(p)=q$ and $f(q)=p$, we can use Statement 55 (see [3, page 242] and [14, pages 97-98]) to conclude that $f$ is an involution on the line $p q$; that is, for any point $x$ on line $p q$, we have $f(f(x))=x$.

In particular, since $f(a)=b$, we have $f(b)=f(f(a))=a$, as required.

\subsection{Some betweenness-only axioms}

One of the reasons for choosing the Klein-Beltrami model of the hyperbolic plane was that its definition of betweenness is lifted from the Cartesian definition of betweenness. Several of Tarski's axioms involve only betweenness, not congruence. Because these axioms have already been verified for the Cartesian plane, the results are often easy to lift to equivalent results in the Klein-Beltrami model. For example, axiom 6 is fairly easily lifted from the Cartesian plane to the Klein-Beltrami model - see Figure 8.4. 


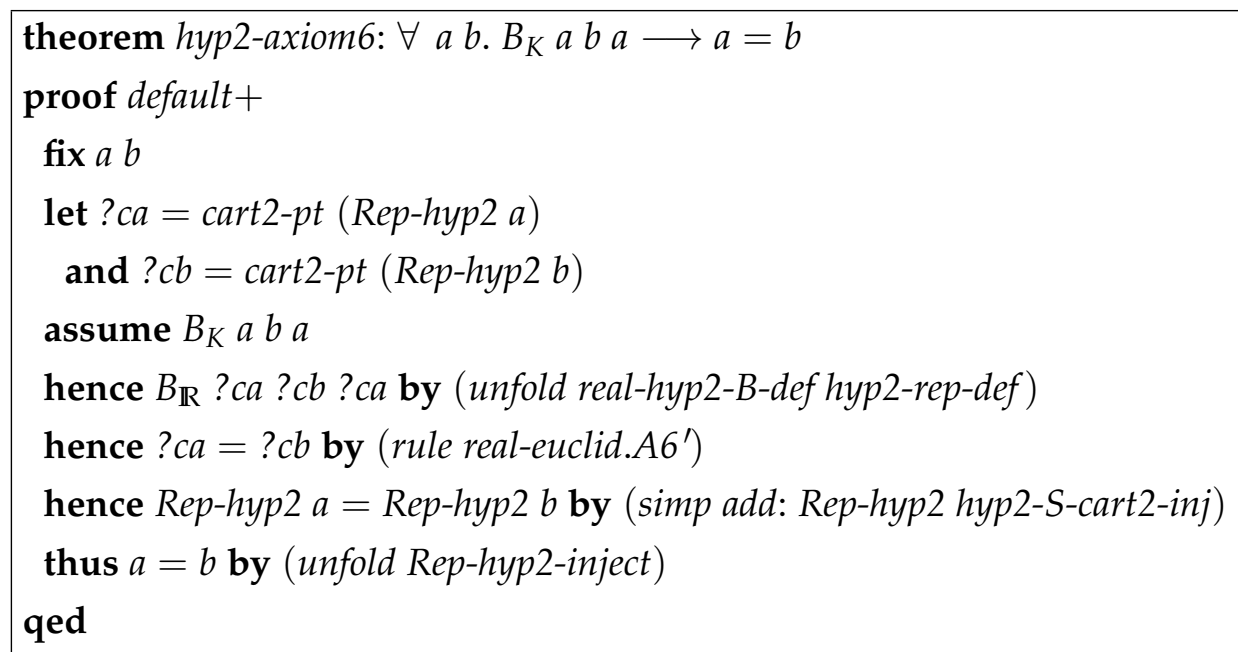

Figure 8.4: Lifting axiom 6 from the Cartesian plane

The attentive reader may notice that Tarski's axiom 10 - the Euclidean axiom - is also a betweenness-only axiom. The question arises as to why this result cannot also be lifted from the Cartesian plane, thus proving that the Klein-Beltrami model does not, in fact, establish the independence of the Euclidean axiom. The answer is that only part of the Cartesian plane corresponds to the points of the hyperbolic plane in the Klein-Beltrami model.

The hypotheses of the Euclidean axiom can be assumed in the KleinBeltrami model, and this configuration does indeed correspond to a similar configuration in the Cartesian plane. The Euclidean axiom in the Cartesian case can then be applied to conclude that the necessary points exist and have the necessary properties. However, the points constructed in this way may fall outside the open unit disc corresponding to the set of points of the hyperbolic plane; it cannot then be concluded that there are points in the Klein-Beltrami model with the necessary properties.

This obstacle also exists for axioms 7, 8, and 11; they all assert that points with certain properties can be constructed. However, for those axioms, the obstacle can be skirted. 
For axiom 7, the point that is constructed is guaranteed to be between two of the given points; because the given points are in the open unit disc, the constructed point must also be in the open unit disc, and can therefore be lifted back to the Klein-Beltrami model — see [14, pages 230-231].

Similarly, for axiom 11 , in the general case the constructed point $b$ is guaranteed to be between a point of $X$ and a point of $Y$. However, the degenerate cases where $X$ or $Y$ is empty must be handled separately; this is dealt with quickly by Isabelle's auto method — see [14, pages 231-232].

For axiom 8, a careful choice of three non-collinear points for the proof in the Cartesian case allowed the same points to be lifted to the KleinBeltrami model. For the formalization, see [14, pages 37-38, 232-233].

\subsection{The axiom of segment construction}

Borsuk and Szmielew's axiom C5 (see [3, page 81]) is similar in spirit to Tarski's axiom of segment construction. Borsuk and Szmielew prove (see [3, pages 255-256]) that the Klein-Beltrami model satisfies their axiom C5. This proof might seem appealing to emulate in order to formally verify that the Klein-Beltrami model satisfies Tarski's axiom of segment construction. However, closer examination reveals that Borsuk and Szmielew's proof relies on a previous statement for which their justification is insufficient.

\subsubsection{Statement 62}

In particular, Borsuk and Szmielew use their Statement 62 (see [3, page 246]) to prove that the Klein-Beltrami model satisfies their axiom C5. Statement 62 asserts that any $K$-isometry maps any open segment of $K_{2}$ to another open segment of $K_{2}$ (with end-points mapped to end-points). The only justification given for Statement 62 is "Since $K$-isometries, as linear transformations, are collineations, then, by Statement 60 , we also 
have [Statement 62]". Statement 60 asserts that $K$-isometries map $K_{2}$ to $K_{2}$.

Nothing in the justification given explains why an open segment in $K_{2}$ might not be mapped to two non-adjacent but collinear open segments in $K_{2}$, for example.

It is possible, given that Statement 62 is about open segments, that Borsuk and Szmielew are implicitly appealing to continuity, as they did explicitly in their terse justification of Statement 61: "Since the K-isometries, as linear transformations, are continuous, then [Statement 61]". In this case, it is not clear what they mean by "continuous".

Although the elliptic plane is essentially equivalent to the projective plane equipped with a metric (and therefore with notions of continuity), Borsuk and Szmielew have not discussed this, except for briefly mentioning the fact in their introduction (see [3, page 6]). Therefore, the reader is left to conclude that Borsuk and Szmielew refer to continuity in the Cartesian plane.

However, it is not the case that all collineations (or even all $\mathrm{K}$-isometries) are continuous in their action on the Cartesian plane. Consider, for example, the action of the collineation represented by

$$
\left(\begin{array}{lll}
0 & 0 & 1 \\
0 & 1 & 0 \\
1 & 0 & 0
\end{array}\right)
$$

on the projective equivalents of the Cartesian points $(\delta, 0)$ and $(-\delta, 0)$, with $\delta>0$. The former is mapped to the projective equivalent of $\left(\frac{1}{\delta}, 0\right)$ and the latter to the equivalent of $\left(\frac{-1}{\delta}, 0\right)$; these can be arbitrarily distant from each other for arbitrarily small $\delta$. In fact, just as the objection above suggested, the open segment with endpoints $(1,0)$ and $(-1,0)$ is mapped to two non-adjacent but collinear open segments, although the point $(0,0)$ is mapped to a projective point that has no Cartesian counterpart according to our fixed (almost-) bijection. 
The collineation in the example above was chosen for clarity, but it is not a $\mathrm{K}$-isometry. However, there are $\mathrm{K}$-isometries that affect the Cartesian plane in similarly discontinuous ways. For example, the collineation represented by

$$
\left(\begin{array}{ccc}
2 & 0 & -1 \\
0 & \sqrt{3} & 0 \\
1 & 0 & -2
\end{array}\right)
$$

is a $\mathrm{K}$-isometry, and given any open segment that crosses the Cartesian line parametrized by $(-2, y)$, this $K$-isometry maps the open segment to two non-adjacent collinear open segments (with the same caveat about one point being mapped to a projective point that has no Cartesian counterpart).

It is now thoroughly demonstrated that Borsuk and Szmielew's justification is insufficient, but the question arises as to whether Statement 62 is true. Although it was not formally verified in Isabelle, I believe that it is true.

It may be argued that although the action of a collineation on the Cartesian plane is not necessarily defined everywhere, it is continuous at all points at which it is defined. This argument is now quite distant from Borsuk and Szmielew's original justification of Statement 62. More importantly, formalizing this argument in Isabelle could be quite complicated; for example, because open segments in $\mathbb{R}^{2}$ are not actually open sets, it may be necessary to associate with each open segment a continuous map from an open interval in $\mathbb{R}$ to the segment, and prove various theorems about these maps.

\subsubsection{Statement 63}

Borsuk and Szmielew's Statement 63, rather than Statement 62, was used in this project's proof that the Klein-Beltrami model satisfies Tarski's axiom 4 . Statement 63 asserts that given a $K$-isometry $f$ and $p, q, r \in$ 
$K_{2} \cup S$ such that B pqr (in the Cartesian plane), it must be the case that B $f(p) f(q) f(r)$. Borsuk and Szmielew's justification of this statement is that it is "an immediate conclusion from" Statement 62 (see [3, page 246]). Therefore, a new proof of Statement 63 was required for the verification in Isabelle of Tarski's axiom 4 . This verification can be found at [14, pages 175-181]; an outline of the proof is given below.

Given a projective point $p \in K_{2} \cup S$, we can be sure that the third coordinate of its representative is not 0 . By dividing a representative in $\mathbb{R}^{3}$ of $p$ by its own third coordinate, we obtain another, standardized representative of $p$, which we can denote by $\bar{p}$, so that $\bar{p}_{3}=1$.

Alternatively, if $\hat{p} \in \mathbb{R}^{2}$ is the Cartesian equivalent of the projective point $p$, we can obtain $\bar{p}$ by appending a 1 to $\widehat{p}$; that is, if $\widehat{p}=(x, y)$, then $\bar{p}=(x, y, 1)$.

Suppose we are given an invertible $3 \times 3$ matrix $J$ that represents a $K$-isometry $f$. If $p \in K_{2} \cup S$, then $\bar{p} J$ represents $f(p)$, which must be in $K_{2} \cup S$, since $f$ is a $K$-isometry. Therefore, $(\bar{p} J)_{3} \neq 0$ and $\bar{p} J=(\bar{p} J)_{3} \overline{f(p)}$.

Given another point $r \in K_{2} \cup S$, suppose that the signs of $(\bar{p} J)_{3}$ and $(\bar{r} J)_{3}$ are opposite. Then let

$$
k=\frac{(\bar{p} J)_{3}}{(\bar{p} J)_{3}-(\bar{r} J)_{3}}
$$

so that $0<k<1$. Let $q$ be the projective point represented by $k \bar{r}+$ $(1-k) \bar{p}$; by considering the third coordinate, we see that this is, in fact, the standardized representative of $q$. Hence $\widehat{q}=k \widehat{r}+(1-k) \widehat{p}$, so B $\widehat{p} \widehat{q} \widehat{r}$. From this, and from the fact that $p, r \in K_{2} \cup S$, we can conclude that $q \in K_{2} \cup S$, so $(\bar{q} J)_{3} \neq 0$. But since $\bar{q}=k \bar{r}+(1-k) \bar{p}$, we have $(\bar{q} J)_{3}=$ $k(\bar{r} J)_{3}+(1-k)(\bar{p} J)_{3}$, which reduces to $(\bar{q} J)_{3}=0$. From this contradiction, we conclude that for any points $p, r \in K_{2} \cup S$, we must have that the signs of $(\bar{p} J)_{3}$ and $(\bar{r} J)_{3}$ are the same.

Now suppose we are given points $p, q$, and $r$ in $K_{2} \cup S$ such that $\mathrm{B} \widehat{p} \widehat{q} \widehat{r}$. Choose $k$ such that $0 \leq k \leq 1$ and $\widehat{q}=k \widehat{r}+(1-k) \hat{p}$. By appending 1 to each of these vectors, we also have that $\bar{q}=k \bar{r}+(1-k) \bar{p}$. Then 


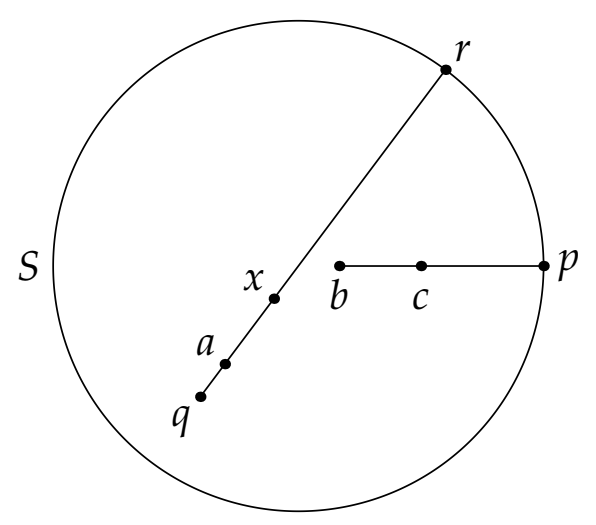

Figure 8.5: Constructions for the verification of axiom 4

$\bar{q} J=k \bar{r} J+(1-k) \bar{p} J$, so that $(\bar{q} J)_{3}=k(\bar{r} J)_{3}+(1-k)(\bar{p} J)_{3}$. Let

$$
c=\frac{k(\bar{r} J)_{3}}{(\bar{q} J)_{3}}
$$

From the previous equation, and because the signs of $(\bar{p} J)_{3},(\bar{q} J)_{3}$, and $(\bar{r} J)_{3}$ are the same, we have $0 \leq c \leq 1$.

From $\bar{q} J=k \bar{r} J+(1-k) \bar{p} J$ we have $(\bar{q} J)_{3} \overline{f(q)}=k(\bar{r} J)_{3} \overline{f(r)}+(1-$ $k)(\bar{p} J)_{3} \overline{f(p)}$. Dividing by $(\bar{q} J)_{3}$, we obtain $\overline{f(q)}=c \overline{f(r)}+(1-c) \overline{f(p)}$. Discarding the third coordinate, we have $\widehat{f(q)}=c \widehat{f(r)}+(1-c) \widehat{f(p)}$, and therefore $\mathrm{B} \widehat{f(p)} \widehat{f(q)} \widehat{f(r)}$. Therefore Statement 63 holds.

\subsubsection{Proof that axiom 4 holds}

To prove that Tarski's axiom 4 holds, assume we are given $a, b, c, q \in K_{2}$, as in Figure 8.5. Extend the segment $b c$ in that direction to intersect $S$ at $p$ (so that $\mathrm{B} \widehat{b} \widehat{c} \widehat{p}$ ), and similarly extend the segment $q a$ in that direction to intersect $S$ at $r$. Using Statement 66, choose a $K$-isometry $f$ such that $f(b)=a$ and $f(p)=r$. Let $x=f(c)$, so that by definition of congruence in the hyperbolic plane, we immediately have $a x \equiv b c$, which is part of what we require. 
From B $\widehat{b} \widehat{c} \widehat{p}$, Statement 63 can be used to conclude that B $\widehat{a} \widehat{x} \widehat{r}$. Together with B $\widehat{q} \widehat{a} \widehat{r}$, this lets us conclude that B $\widehat{q} \widehat{a} \widehat{x}$, by part of Satz 3.5 (see [21, page 30] and [14, page 23]). By the definition of betweenness in the hyperbolic plane, we have established all that is required.

\subsection{The five-segments axiom}

Borsuk and Szmielew give a simple proof in [3, page 256] that the KleinBeltrami model satisfies the five-segments axiom. Although this proof is relatively easy to formalize, it depends on a lemma - Borsuk and Szmielew's Statement 69 - for which their proof is long and messy.

Statement 69 says that if we are given $a, b, c, a^{\prime}, b^{\prime}, c^{\prime} \in K_{2}$ such that $a b \equiv a^{\prime} b^{\prime}, b c \equiv b^{\prime} c^{\prime}$, and $a c \equiv a^{\prime} c^{\prime}$ (in the hyperbolic plane), then there is a $K$-isometry $f$ such that $f(a)=a^{\prime}, f(b)=b^{\prime}$, and $f(c)=c^{\prime}$. The first two hypotheses alone assure us that there are $K$-isometries $f$ and $g$ such that $f(a)=a^{\prime}, f(b)=b^{\prime}, g(b)=b^{\prime}$, and $g(c)=c^{\prime}$, but we seek a single $K$-isometry that maps $a, b$, and $c$ to $a^{\prime}, b^{\prime}$, and $c^{\prime}$, respectively.

Borsuk and Szmielew's proof of Statement 69 (see [3, pages 251-255]) is quite long, making it daunting to write an inevitably longer formalization. In their nearly four-page proof, they consider in detail only the case where segment $a b$ is shorter than segment $a c$ (after assuming, without loss of generality, that $a b$ is no longer than $a c)$; for the case where $a b \equiv a c$, although they give a diagram, they say only that "The argument is analogous, but simpler, since [various points] do not enter into it".

Further complicating a possible formalization is the fact that Borsuk and Szmielew's proof involves half-lines, half-planes, and a betweenness relation on half-lines in the Cartesian plane. Because these are not needed for the verification of any of Tarski's other axioms, the formalization of these concepts can be added to the cost of formalizing Borsuk and Szmielew's proof of Statement 69.

For these reasons, a new proof of Statement 69 was sought - and 
found. This new proof is not without its own complications. It required the formalization of what turns out to be perpendicularity in the model of the hyperbolic plane, as well as formalization of cross ratios in the projective plane; it even came very close to formalizing a distance function on the points of the hyperbolic plane. However, many of the definitions and lemmas required to prove Statement 69 were also useful in the verification that the Klein-Beltrami model satisfies Tarski's axiom 9 - see Section 8.6.

\subsubsection{Perpendicularity}

Perpendicularity in the hyperbolic plane can be characterized in the KleinBeltrami model as follows. Given a line $l$ that passes through $K_{2}$, let $p$ and $q$ denote the intersections of $l$ with $S$. Let $r$ denote the intersection of the tangents to $S$ at $p$ and $q$; this is the pole of $l$. The lines through $r$ that pass through $K_{2}$ are the lines perpendicular to $l$.

A right angle in the Klein-Beltrami model can be defined to consist of three points, $p, a$, and $q$, such that $p$ and $q$ are in $S, a$ is in $K_{2}$, and the lines $p a$ and $a q$ are perpendicular.

The constructions used in the definition of perpendicularity are similar to those shown in Figure 8.2. In fact, by using arguments similar to those in Subsection 8.2.2, it can be shown that any right angle can be mapped to any other right angle by a K-isometry.

See [14, pages 190-203] for the full formalization of perpendicularity and right angles.

\subsubsection{Cross ratios}

The cross ratio is a function that takes four collinear projective points as its arguments; its values are taken from $\mathbb{R}$. The cross ratio of $p, q, r$, and $s$ is denoted $(p, q ; r, s)$. This function has several useful properties; for example: 
- collineations preserve cross ratios;

- if $p, q$, and $r$ are distinct and collinear, then the value of $(p, q ; r, s)$ uniquely determines the point $s$ on the line $p q$;

- if $p$ and $q$ are distinct, and $r, s$, and $t$ are collinear with, but distinct from, $p$ and $q$, then $(p, q ; r, s)(p, q ; s, t)=(p, q ; r, t)$;

- in particular, $(p, q ; r, r)=1$ and

$$
(p, q ; r, s)=\frac{1}{(p, q ; s, r)}
$$

For more on cross ratios, see [3, pages 235-238] and [14, pages 98-106].

\subsubsection{Distance}

Given points $a$ and $b$ in $K_{2}$, let $p$ and $q$ denote the intersections of the line $a b$ with the absolute $S$ (if $a=b$, then any line through $a$ will do). We can define the distance between the hyperbolic points represented by $a$ and $b$ to be $\rho(a, b)=\frac{1}{2}|\log (p, q ; a, b)|$, where $\log$ denotes the natural logarithm.

In Section 5.3, when verifying that the Cartesian plane satisfies Tarski's five-segments axiom, we found it convenient to use the cosine of an angle, rather than formalizing angles themselves. Similarly, when verifying that the Klein-Beltrami model satisfies Tarski's five-segments axiom, it is more convenient to use the hyperbolic cosine of segment lengths, rather than formalizing lengths themselves. For convenience, this simplifies to

$$
\cosh (\rho(a, b))=\frac{\sqrt{(p, q ; a, b)}+\sqrt{(p, q ; b, a)}}{2}
$$

$K$-isometries, as collineations, preserve cross ratios; given a line $l$, a $K$-isometry will also map the intersections of $S$ with $l$ to the intersections of $S$ with the image of $l$. Consequently, $K$-isometries must preserve the above function of distance. Therefore, by the definition of congruence, if $a b \equiv a^{\prime} b^{\prime}$, then $\cosh (\rho(a, b))=\cosh \left(\rho\left(a^{\prime}, b^{\prime}\right)\right)$. 
Among the theorems about this function of distance, a notable one is a formula for $\cosh (\rho(a, b))$ in terms of representatives of $a$ and $b$. Given any representatives $\tilde{a}$ and $\tilde{b}$ of the projective points $a$ and $b$ (which in turn represent hyperbolic points),

$$
\cosh (\rho(a, b))=\frac{\left|\tilde{a} M \tilde{b}^{\top}\right|}{\sqrt{\tilde{a} M \tilde{a}^{\top} \tilde{b} M \tilde{b}^{\top}}}
$$

By recalling our choice of $M$, and by using the standardized representatives $\tilde{a}=\bar{a}$ and $\tilde{b}=\bar{b}$, it can be seen that

$$
(\cosh (\rho(a, b)))^{2}=\frac{(1-\widehat{a} \cdot \widehat{b})^{2}}{\left(1-\|\widehat{a}\|^{2}\right)\left(1-\|\widehat{b}\|^{2}\right)}
$$

This is clearly suggestive of the model in [21, page 208], mentioned above in Section 6.2.

Recall that this model was avoided because of the lack of published proofs that it satisfies Tarski's axioms, including the five-segments axiom; instead, Borsuk and Szmielew's Klein-Beltrami model was chosen because they did verify that it satisfies the five-segments axiom. Now, when it comes to formalizing their proof, it is judged to be so difficult that an alternative proof is sought; the alternative proof that was found takes us strikingly close to the model we tried to avoid.

It would be interesting to know whether verifying Tarski's axioms would be easier if we started with the model in [21, page 208] and tried to avoid having to formalize projective geometry. It may be the case that an attempt to do so would involve considering numerous degenerate cases where lines are parallel in the Cartesian plane; such an attempt may lead to the conclusion that it is simpler to use the projective plane to define the model of the hyperbolic plane.

Another useful theorem about distance in the hyperbolic plane is the hyperbolic equivalent of the so-called theorem of Pythagoras: if $a, b$, and $c$ are the vertices of a right-angled triangle, with the right angle at $b$, then $\cosh (\rho(a, c))=\cosh (\rho(b, a)) \cosh (\rho(b, c))$. 
For the formalization of the concepts discussed in this subsection, see [14, pages 203-220].

\subsubsection{A formula for a cross ratio involving a perpendicular foot}

Suppose we are given points $a, b$, and $c$ in the Klein-Beltrami model, with $a \neq b$. Let $d$ denote the perpendicular foot of $c$ on line $a b$. Then

$$
\begin{aligned}
& \cosh (\rho(a, c))=\cosh (\rho(d, a)) \cosh (\rho(d, c)) \quad \text { and } \\
& \cosh (\rho(b, c))=\cosh (\rho(d, b)) \cosh (\rho(d, c))
\end{aligned}
$$

Dividing the first equation by the second gives us

$$
\frac{\cosh (\rho(a, c))}{\cosh (\rho(b, c))}=\frac{\cosh (\rho(d, a))}{\cosh (\rho(d, b))}
$$

If $p$ and $q$ are the intersections of line $a b$ with $S$, then

$$
\frac{\cosh (\rho(a, c))}{\cosh (\rho(b, c))}=\frac{\sqrt{(p, q ; d, a)}+\sqrt{(p, q ; a, d)}}{\sqrt{(p, q ; d, b)}+\sqrt{(p, q ; b, d)}}
$$

Multiplying the top and bottom of the right-hand side by $\sqrt{(p, q ; d, b)}$, and recalling some properties of cross ratios - particularly $(p, q ; d, b)=$ $(p, q ; d, a)(p, q ; a, b)$ - we obtain

$$
\frac{\cosh (\rho(a, c))}{\cosh (\rho(b, c))}=\frac{(p, q ; d, a) \sqrt{(p, q ; a, b)}+\sqrt{(p, q ; a, b)}}{(p, q ; d, a)(p, q ; a, b)+1}
$$

Rearranging for $(p, q ; d, a)$ yields

$$
(p, q ; d, a)=\frac{\cosh (\rho(b, c)) \sqrt{(p, q ; a, b)}-\cosh (\rho(a, c))}{\cosh (\rho(a, c))(p, q ; a, b)-\cosh (\rho(b, c)) \sqrt{(p, q ; a, b)}}
$$

Although this formula is somewhat messy, it establishes the cross ratio involving the perpendicular foot $d$ in terms of cross ratios that do not involve $d$.

The formalization of this formula can be found at [14, pages 220-223]. 


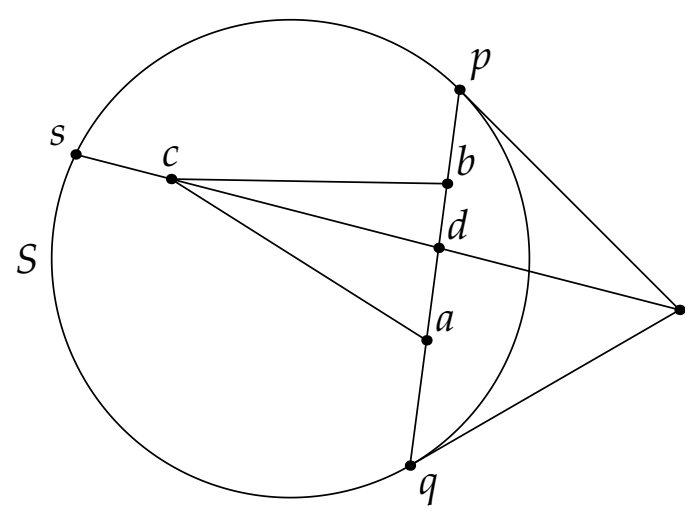

Figure 8.6: Constructions for the proof of Statement 69

\subsubsection{Proof that Statement 69 and axiom 5 hold}

To prove that Statement 69 holds, a degenerate case is first considered. If $a=b$, then because $a b \equiv a^{\prime} b^{\prime}$, it can be shown that $a^{\prime}=b^{\prime}$. By the hypothesis that $b c \equiv b^{\prime} c^{\prime}$, choose a K-isometry $f$ such that $f(b)=b^{\prime}$ and $f(c)=c^{\prime}$. Furthermore, $f(a)=f(b)=b^{\prime}=a^{\prime}$, so $f$ is the $K$-isometry required.

Suppose instead that $a \neq b$. We perform some constructions shown in Figure 8.6. First, segment $a b$ is extended in that direction to intersect $S$ at $p$. Let $q$ denote the other intersection of line $a b$ with $S$. Let $d$ denote the foot of the perpendicular dropped from $c$ to the line $p q$. Extend segment $d c$ in that direction to intersect $S$ at $s$.

These same constructions are performed on $a^{\prime}, b^{\prime}$, and $c^{\prime}$, to obtain $p^{\prime}$, $q^{\prime}, d^{\prime}$, and $s^{\prime}$

A $K$-isometry $f$ is chosen to map right angle $p d s$ to right angle $p^{\prime} d^{\prime} s^{\prime}$.

Both $f(q)$ and $q^{\prime}$ are in $S$ on the line $p^{\prime} d^{\prime}$, and neither can coincide with $f(p)=p^{\prime}$. Because there are only two intersections of line $p^{\prime} d^{\prime}$ with $S$, and we have ruled out $p^{\prime}$, we must have $f(q)=q^{\prime}$.

From $a b \equiv a^{\prime} b^{\prime}$ we can establish that $(p, q ; a, b)=\left(p^{\prime}, q^{\prime} ; a^{\prime}, b^{\prime}\right)$. Also, from $b c \equiv b^{\prime} c^{\prime}$ and $a c \equiv a^{\prime} c^{\prime}$ we have $\cosh (\rho(b, c))=\cosh \left(\rho\left(b^{\prime}, c^{\prime}\right)\right)$ and 
$\cosh (\rho(a, c))=\cosh \left(\rho\left(a^{\prime}, c^{\prime}\right)\right)$. Therefore, using the formula derived in Subsection 8.5.4, we can conclude that $(p, q ; d, a)=\left(p^{\prime}, q^{\prime} ; d^{\prime}, a^{\prime}\right)$. But because collineations preserve cross ratios, we also have $(p, q ; d, a)=$ $(f(p), f(q) ; f(d), f(a))=\left(p^{\prime}, q^{\prime} ; d^{\prime}, f(a)\right)$. The uniqueness property of cross ratios then tells us that $f(a)=a^{\prime}$.

Similarly, because we have $(p, q ; a, b)=\left(p^{\prime}, q^{\prime} ; a^{\prime}, b^{\prime}\right)$ and now also $(p, q ; a, b)=\left(p^{\prime}, q^{\prime} ; a^{\prime}, f(b)\right)$, we can conclude that $f(b)=b^{\prime}$.

Because $f(c)$ and $c^{\prime}$ both lie on line $s^{\prime} d^{\prime}$, and line $a^{\prime} d^{\prime}$ is perpendicular, we have

$$
\begin{aligned}
\cosh \left(\rho\left(a^{\prime}, f(c)\right)\right) & =\cosh \left(\rho\left(d^{\prime}, a^{\prime}\right)\right) \cosh \left(\rho\left(d^{\prime}, f(c)\right)\right) \quad \text { and } \\
\cosh \left(\rho\left(a^{\prime}, c^{\prime}\right)\right) & =\cosh \left(\rho\left(d^{\prime}, a^{\prime}\right)\right) \cosh \left(\rho\left(d^{\prime}, c^{\prime}\right)\right)
\end{aligned}
$$

But we already have $\cosh (\rho(a, c))=\cosh \left(\rho\left(a^{\prime}, c^{\prime}\right)\right)$, and because $f$ is a $K$ isometry we have $\cosh (\rho(a, c))=\cosh (\rho(f(a), f(c)))=\cosh \left(\rho\left(a^{\prime}, f(c)\right)\right)$. All together, these let us conclude that $\cosh \left(\rho\left(d^{\prime}, f(c)\right)\right)=\cosh \left(\rho\left(d^{\prime}, c^{\prime}\right)\right)$.

There is a theorem that lets us conclude, from B $\widehat{d^{\prime}} \widehat{f(c)} \widehat{s^{\prime}}, \mathrm{B} \widehat{d^{\prime}} \widehat{c^{\prime}} \widehat{s^{\prime}}$, and $\cosh \left(\rho\left(d^{\prime}, f(c)\right)\right)=\cosh \left(\rho\left(d^{\prime}, c^{\prime}\right)\right)$, that we must have $f(c)=c^{\prime}$. (Essentially, the theorem ensures that if we fix $x$, the direction of $x y$, and the value of $\cosh (\rho(x, y))$, then we have fixed the point $y$.)

We have finally shown that our choice of $f$ - which initially fixed only $f(p)=p^{\prime}, f(d)=d^{\prime}$, and $f(s)=s^{\prime}$ - must also fix $f(a)=a^{\prime}, f(b)=b^{\prime}$, and $f(c)=c^{\prime}$. The existence of such a $K$-isometry proves Statement 69 .

Now, to prove that axiom 5 holds, suppose we have B $\widehat{a} \widehat{b} \widehat{c}, \mathrm{~B} \widehat{a^{\prime}} \widehat{b}^{\prime} \widehat{c}^{\prime}$, $a b \equiv a^{\prime} b^{\prime}, b c \equiv b^{\prime} c^{\prime}, a d \equiv a^{\prime} d^{\prime}, b d \equiv b^{\prime} d^{\prime}$, and $a \neq b$.

From $a b \equiv a^{\prime} b^{\prime}, b d \equiv b^{\prime} d^{\prime}$, and $a d \equiv a^{\prime} d^{\prime}$, we can use Statement 69 to choose a K-isometry such that $f(a)=a^{\prime}, f(b)=b^{\prime}$, and $f(d)=d^{\prime}$.

Now B $\widehat{a} \widehat{b} \widehat{c}$ gives us B $\widehat{a^{\prime}} \widehat{b^{\prime}} \widehat{f(c)}$, and we already have B $\widehat{a^{\prime}} \widehat{b^{\prime}} \widehat{c^{\prime}}$. Because $a \neq b$ (and therefore $a^{\prime} \neq b^{\prime}$ ), this fixes the direction of $b^{\prime} f(c)$ to be the same as the direction of $b^{\prime} c^{\prime}$. Because $b c \equiv b^{\prime} c^{\prime}$, this fixes $\cosh \left(\rho\left(b^{\prime}, f(c)\right)\right)=$ $\cosh \left(\rho\left(b^{\prime}, c^{\prime}\right)\right)$ also. Therefore, we must have $f(c)=c^{\prime}$.

With $f(c)=c^{\prime}$ and $f(d)=d^{\prime}$, the definition of congruence lets us 
conclude $c d \equiv c^{\prime} d^{\prime}$, as required.

The proofs discussed in this subsection are formalized in Isabelle in [14, pages 218-219, 223-229].

\subsection{The upper 2-dimensional axiom}

\subsubsection{Non-categoricity of Borsuk and Szmielew's axioms}

Given that the model that we are using was taken from [3], it is natural to look there for a proof of something similar to Tarski's axiom 9; after all, we found proofs there of theorems similar to axioms 1,4, and 5, although they were of varying helpfulness.

One encouragement is that Borsuk and Szmielew conclude Part One of their book with Proposition 7, which states that "The axiom system $\left(\mathrm{GBL}_{2}\right)$ of plane Lobachevskian [that is, hyperbolic] geometry is categorical" - see [3, pages 344-345]. If this is true, then their axioms must somehow restrict the geometry to two dimensions. It may be the case that for this purpose they use an axiom similar to Tarski's axiom 9; if so, then it is probably worthwhile consulting their proof that the KleinBeltrami model satisfies this axiom. However, closer inspection of their axiom system $\left(\mathrm{GBL}_{2}\right)$ reveals that it does not, in fact, restrict geometry to two dimensions, and that Proposition 7 must be false, although it may be possible to repair it without much disruption to Borsuk and Szmielew's argument.

Borsuk and Szmielew discuss their various axioms in [3, pages 196197]. The axiom system $\left(\mathrm{GA}_{3}\right)$ of 3-dimensional absolute geometry consists of nine axioms of incidence, I1-I9, nine axioms of order, O1-O9, seven axioms of congruence, $\mathrm{C} 1-\mathrm{C} 7$, and one axiom of continuity, Co. One model of $\left(\mathrm{GA}_{3}\right)$ is 3-dimensional real Cartesian space, $\mathbb{R}^{3}$.

The axiom system $\left(\mathrm{GA}_{2}\right)$ of absolute plane geometry consists of axioms I1-I4, O1-O9, C1-C7, and Co, omitting axioms I5-I9. In this system, 
axiom I4 is weakened to assert only the existence of three non-collinear points, rather than that every plane has three non-collinear points. It is clear then that any model of $\left(\mathrm{GA}_{3}\right)$ is also a model of $\left(\mathrm{GA}_{2}\right)$; in particular, $\mathbb{R}^{3}$ is a model of $\left(\mathrm{GA}_{2}\right)$.

In [3, page 344], the axiom system $\left(\mathrm{GBL}_{2}\right)$ is defined to consist of the axioms of $\left(\mathrm{GA}_{2}\right)$ together with a non-Euclidean axiom $\mathrm{BL}$, which is essentially the negation of Playfair's axiom. But in $\mathbb{R}^{3}$, given a line $l$ and a point $P$ not on $l$, many lines pass through $P$ that do not intersect $l$ - any line through $P$ that does not lie in a plane with $l$, for example. Therefore $\mathbb{R}^{3}$ is a model of $\left(\mathrm{GBL}_{2}\right)$. Because the Klein-Beltrami model is also a model of $\left(\mathrm{GBL}_{2}\right)$ - and is not isomorphic to $\mathbb{R}^{3}$ (because $\mathbb{R}^{3}$ does not satisfy Tarski's upper 2-dimensional axiom) - we see that $\left(\mathrm{GBL}_{2}\right)$ is not categorical and Proposition 7 is false.

This situation may be remedied by altering axiom $\mathrm{O} 9$ in the systems $\left(\mathrm{GA}_{2}\right)$ and $\left(\mathrm{GBL}_{2}\right)$. Axiom $\mathrm{O} 9$ is defined in [3, page 42] and is a version of the axiom of Pasch. It is called the "plane axiom of order" and its hypotheses explicitly restrict it to a plane. If we remove this restriction to a plane in the hypotheses, then it may force the whole geometry to lie in a single plane. This would need to be checked carefully. In any case, it is not similar to Tarski's axiom 9, so any proof that the Klein-Beltrami model satisfies this stricter axiom $\mathrm{O} 9$ may not be useful in proving that it satisfies Tarski's axiom 9. Therefore, we require another proof that the Klein-Beltrami model satisfies Tarski's axiom 9.

\subsubsection{Proof that axiom 9 holds}

In the proof that axiom 9 holds in the Klein-Beltrami model, we re-use much of the infrastructure that was developed for the proof of axiom 5.

Suppose $p$ and $q$ are distinct points in $K_{2}$. Extend segment $p q$ in that direction to intersect $S$ at $s$ (see Figure 8.7). Let $t$ be the other intersection of line $p q$ with $S$. Let $r$ be the intersection of the tangents to $S$ at $s$ and $t$. 


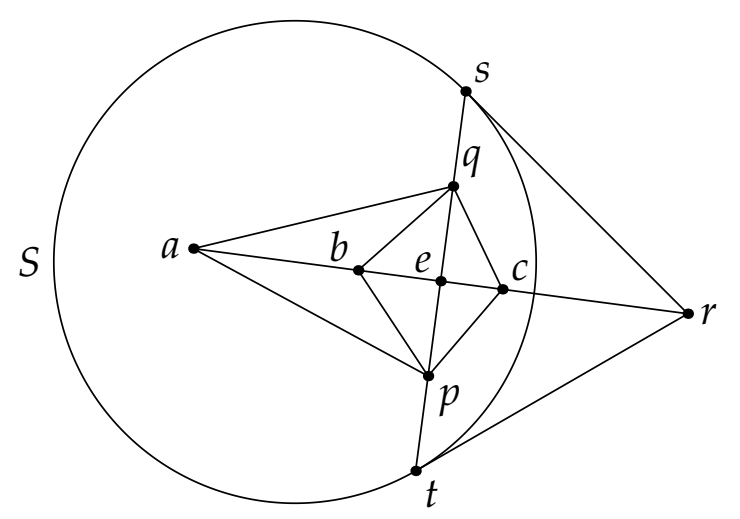

Figure 8.7: Constructions for the verification of axiom 9

Suppose we have a point $d \in K_{2}$ such that $d p \equiv d q$. Let $e$ denote the perpendicular foot of $d$ on line $s t$; that is, let $e$ be the intersection of line $d r$ and line st. According to the formula derived in Subsection 8.5.4,

$$
(s, t ; e, p)=\frac{\cosh (\rho(q, d)) \sqrt{(s, t ; p, q)}-\cosh (\rho(p, d))}{\cosh (\rho(p, d))(s, t ; p, q)-\cosh (\rho(q, d)) \sqrt{(s, t ; p, q)}}
$$

Because $d p \equiv d q$, we have $\cosh (\rho(p, d))=\cosh (\rho(q, d))$, so the above simplifies to

$$
(s, t ; e, p)=\frac{1}{\sqrt{(s, t ; p, q)}}
$$

or $(s, t ; p, e)=\sqrt{(s, t ; p, q)}$.

Now, suppose we have points $a, b$, and $c$ in $K_{2}$ such that $a p \equiv a q$, $b p \equiv b q$, and $c p \equiv c q$. The above, together with the uniqueness property of cross ratios, lets us conclude that the perpendicular feet of $a, b$, and $c$ on line st all coincide; let $e$ denote this perpendicular foot.

Now, by the definition of perpendicular foot, we know that $a$ lies on the line er. Similarly, $b$ and $c$ also lie on the line er. Because $e \in K_{2}$ and $r \notin K_{2}$, we know that $e$ and $r$ are distinct, so we can conclude that $a, b$, and $c$ are collinear. This is sufficient to prove that $\mathrm{B} a b c \vee \mathrm{B} b c a \vee \mathrm{B} c a b$, as required.

The Isabelle formalization of this proof is in [14, pages 233-235]. 
interpretation hyp2: tarski-absolute real-hyp2-C real-hyp2-B

using hyp2-axiom 8 and hyp2-axiom 9

by unfold-locales

Figure 8.8: The model satisfies the axioms of absolute plane geometry

\subsection{The negation of the Euclidean axiom}

We have now discussed the proofs that the Klein-Beltrami model satisfies each of Tarski's axioms except the Euclidean axiom. This result is summarized by the Isabelle extract shown in Figure 8.8 - recall that the locale tarski-absolute embodies all of Tarski's axioms except the Euclidean axiom. The Isabelle proof merely recalls the already-verified theorems that axioms 8 and 9 hold; none of the other axioms are mentioned because it has already been verified that the Klein-Beltrami model satisfies tarski-absolute-space.

All that is left is to check whether the Klein-Beltrami model satisfies the Euclidean axiom. If it does satisfy the Euclidean axiom, then either Tarski's axioms are not actually categorical, or the Klein-Beltrami model is a very elaborate model of the Euclidean plane.

In fact, Tarski's Euclidean axiom does not hold in the Klein-Beltrami model. For a counter-example, we can take $a, b, c, d$, and $t$ to be the projective equivalents of $(0,0),\left(\frac{1}{2}, 0\right),\left(0, \frac{1}{2}\right),\left(\frac{1}{4}, \frac{1}{4}\right)$, and $\left(\frac{1}{2}, \frac{1}{2}\right)$, respectively, according to our fixed bijection - see Figure 8.9. It is then relatively straightforward to check that these points are in $K_{2}$ (and therefore represent points of the hyperbolic plane) and that they satisfy the hypotheses of Tarski's Euclidean axiom.

It is also not difficult to prove that the conclusion of axiom 10 does not hold. Consider a line through $t$ that intersects line $a b$ at $x$ and line $a c$ at $y$. In order to ensure B $\widehat{a} \widehat{b} \widehat{x}$ and B $\widehat{a} \widehat{c} \widehat{y}$, we must have either $x$ or $y$ (or both) outside $K_{2}$ (although, as Figure 8.9 suggests, it may be that $x$ and $y$ are both in $S$, which is disjoint from $K_{2}$ ). Therefore there can be no suitable 


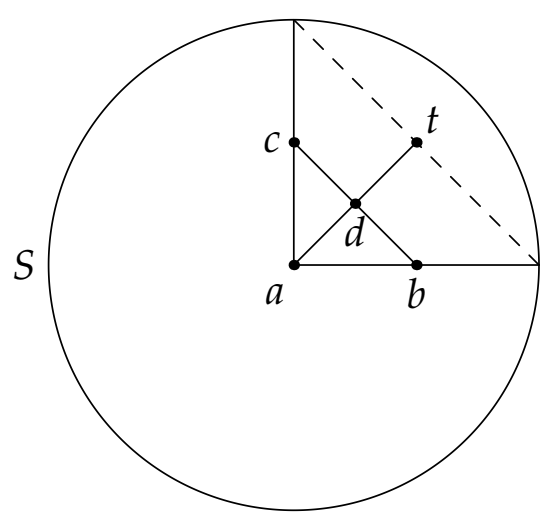

Figure 8.9: A counter-example to the Euclidean axiom

theorem hyp2-not-tarski: $\neg($ tarski real-hyp2-C real-hyp2-B)

using hyp2-axiom10-false

by (unfold tarski-def tarski-space-def tarski-space-axioms-def) simp

Figure 8.10: The model does not satisfy all of the axioms

points $x$ and $y$ that represent points of the hyperbolic plane.

The Isabelle formalization of this counter-example can be found in [14, pages 235-237]. The Isabelle extract in Figure 8.10 is the final demonstration that what we showed in Figure 8.8 is a model of tarski-absolute is not also a model of tarski.

This concludes the verification that Tarski's Euclidean axiom is independent of his other axioms of the Euclidean plane. 


\section{Chapter 9}

\section{Conclusion}

\subsection{Success}

The initial goal of this project was to mechanically verify the independence of the parallels postulate. In order to make this verification as meaningful as possible, Tarski's axiom system for plane geometry was chosen - in part because it is categorical.

Tarski's axioms were successfully formalized in the proof verification program Isabelle. A model was provided for the axioms, establishing that they are consistent. Then, the Klein-Beltrami model of the hyperbolic plane was formalized and shown to be a model of all of Tarski's axioms except the Euclidean axiom, which it violates. This established that Tarski's Euclidean axiom is independent of Tarski's other axioms of plane geometry.

Along the way, the projective plane was also formalized, and many theorems were proven about it. Some published proofs about a model of the hyperbolic plane were questioned, and alternative proofs were found and verified. 


\subsection{Equivalent axioms}

This success is not quite the whole story, if the goal was to verify the independence of the parallels postulate; although Tarski's Euclidean axiom is equivalent to Euclid's original parallels postulate, it is not obviously so.

Therefore, a natural extension of the work described in this thesis would be to mechanically verify that - in the context of Tarski's other axioms - Tarski's Euclidean axiom is equivalent to Euclid's parallels postulate. This would require the formalization of concepts in and consequences of Tarski's axioms of absolute plane geometry; this thesis was primarily concerned with models, rather than consequences, of Tarski's axioms.

In order even to state Euclid's parallels postulate in the context of Tarski's axioms, several notions would first need to be defined - for example: lines, intersection of lines, angles, addition of angles, and what it means for an angle to be on a particular side of a line. As a first step towards this goal (and as a worthy goal in itself), it may be worthwhile first proving that Playfair's axiom is equivalent to Tarski's Euclidean axiom. The statement of Playfair's axiom would require the formalization only of lines, intersection of lines, and parallelism of lines.

For the formalization within Tarski's axiom system of concepts such as lines and angles, a useful resource would be [21] — particularly Teil I. Also of likely usefulness would be Narboux's existing work [16] on formalizing in Coq some parts of [21].

\subsection{Other possible future work}

A possible complement to the work described in this thesis would be mechanically verified proofs of the independence of some of Tarski's other axioms. Two of these independence proofs - those relating to the lower and upper 2-dimensional axioms - could be completed relatively swiftly, 
since, as Section 5.1 mentions, all of the other axioms have already been verified for $\mathbb{R}^{n}$.

Another metamathematical result relating to Tarski's axioms is the categoricity of the axiom system; this result would also be nice to have formally verified. Also, similar independence and categoricity questions arise when Tarski's Euclidean axiom is replaced with a hyperbolic axiom, as in [21, page 204]. Answers to at least some of these questions are known; these answers, too, would be interesting to have mechanically verified.

Finally, as was briefly discussed in Subsection 8.5.3, it may be interesting to know whether it would be easier to avoid the projective plane in the proof of the independence of Tarski's Euclidean axiom. If it turns out to be easier to avoid the projective plane, it is hoped that this project's mechanically verified theory of the projective plane is considered worthwhile in its own right. 


\section{Bibliography}

[1] Behnke, H., Bachmann, F., Fladt, K., and Kunle, H., Eds. Fundamentals of Mathematics, vol. 2, Geometry. The Massachusetts Institute of Technology, 1974. Translated by S. H. Gould.

[2] Beltrami, E. Teoria fondamentale degli spazii di curvatura costante. Annali di Matematica Pura ed Applicata (1867-1897) 2, 1 (1868), 232255.

[3] Borsuk, K., And Szmielew, W. Foundations of Geometry: Euclidean and Bolyai-Lobachevskian Geometry; Projective Geometry. North-Holland Publishing Company, 1960. Translated from Polish by Erwin Marquit.

[4] Chou, S.-C., GaO, X.-S., And Zhang, J.-Z. Area method and automated reasoning in affine geometries. Accessed November 2011 from http://www.cs.wichita.edu/ chou/pdf/t-aff .pdf.

[5] Coxeter, H. S. M. Non-Euclidean Geometry, second ed. No. 2 in Mathematical Expositions. The University of Toronto Press, 1947.

[6] Dehlinger, C., Dufourd, J.-F., And Schreck, P. Higher-order intuitionistic formalization and proofs in Hilbert's elementary geometry. In Automated Deduction in Geometry, J. Richter-Gebert and D. Wang, Eds., vol. 2061 of Lecture Notes in Computer Science. Springer Berlin / Heidelberg, 2001, pp. 306-323. 
[7] Euclid, and Heath, T. L. The Thirteen Books of Euclid's Elements: Translated from the Text of Heiberg with Introduction and Commentary by T. L. Heath, vol. 1. Cambridge University Press, 1908.

[8] Euclid, and Playfair, J. Elements of Geometry: Containing the First Six Books of Euclid, with a Supplement on the Quadrature of the Circle and the Geometry of Solids; to which are added Elements of Plane and Spherical Trigonometry. George Long, 1819.

[9] Fleuriot, J. A Combination of Geometry Theorem Proving and Nonstandard Analysis with Application to Newton's Principia. Springer, 2001.

[10] Forder, H. G. The Foundations of Euclidean Geometry. Cambridge University Press, 1927.

[11] Henkin, L., Suppes, P., and Tarski, A., Eds. The Axiomatic Method: with Special Reference to Geometry and Physics (1959), North-Holland Publishing Company.

[12] Hilbert, D. The Foundations of Geometry. Project Gutenberg, 2005. Translated by E. J. Townsend. http://www.gutenberg. org/ebooks/ 17384.

[13] Klein, G., Nipkow, T., and Paulson, L., Eds. The Archive of Formal Proofs. http://afp. sourceforge.net/.

[14] Makarios, T. J. M. The independence of the parallels postulate, verified in Isabelle 2009-2. This typeset Isabelle source should be available with this thesis, 2012.

[15] Meikle, L. I., And Fleuriot, J. D. Formalizing Hilbert's Grundlagen in Isabelle/Isar. In Theorem Proving in Higher Order Logics, D. Basin and B. Wolff, Eds., vol. 2758 of Lecture Notes in Computer Science. Springer Berlin / Heidelberg, 2003, pp. 319-334. 
[16] Narboux, J. Mechanical theorem proving in Tarski's geometry. In Automated Deduction in Geometry, F. Botana and T. Recio, Eds., vol. 4869 of Lecture Notes in Computer Science. Springer Berlin / Heidelberg, 2007, pp. 139-156.

[17] Newton, I. Philosophiz Naturalis Principia Mathematica. Edmond Halley, 1687.

[18] Pambuccian, V. Axiomatizations of hyperbolic geometry: A comparison based on language and quantifier type complexity. Synthese 133 (2002), 331-341.

[19] PAsch, M. Vorlesungen über neuere Geometrie. B. G. Teubner, 1882.

[20] Rosenfeld, B. A. A History of Non-Euclidean Geometry: Evolution of the Concept of a Geometric Space. Springer-Verlag, 1988. Translated from Russian by A. Shenitzer.

[21] SchwabhëUser, W., Szmielew, W., ANd Tarski, A. Metamathematische Methoden in der Geometrie. Springer-Verlag, 1983.

[22] Sсотт, P. Mechanising Hilbert's Foundations of Geometry in Isabelle. Master's thesis, University of Edinburgh, 2008. http://www .inf .ed. ac.uk/publications/thesis/online/IM080566.pdf.

[23] Sмiтh, D. E. History of Mathematics, vol. 1. Dover, 1958.

[24] Stillwell, J. Mathematics and Its History. Springer-Verlag, 1989.

[25] Stump, D. J. The independence of the parallel postulate and development of rigorous consistency proofs. History and Philosophy of Logic 28, 1 (2007), 19-30.

[26] Szmielew, W. Some metamathematical problems concerning elementary hyperbolic geometry. In Henkin et al. [11], pp. 30-52. 
[27] TARsKi, A. A decision method for elementary algebra and geometry. Tech. rep., The RAND Corporation, 1957. First published August 1948.

[28] TARSKI, A. What is elementary geometry? In Henkin et al. [11], pp. 16-29.

[29] TARsKI, A., AND Givant, S. Tarski's system of geometry. The Bulletin of Symbolic Logic 5, 2 (June 1999), 175-214.

[30] Tezzaron Semiconductor. Soft errors in electronic memory - a white paper. http://www.tezzaron.com/about/papers/soft errors_1_1_secure.pdf, January 2004.

[31] Urban, J. PVS GPLed, 2008. http://old.nabble.com/ TPHOLs-becomes-ITP-\%28f wd\%29-tt19435554 . html\#a19449964.

[32] Veblen, O., and Young, J. W. Projective Geometry, vol. 2. Ginn and Company, 1918.

[33] WiedijK, F., Ed. The Seventeen Provers of the World. 2005. http: //www.cs.ru.nl/ freek/comparison/comparison.pdf.

[34] WiEDIJK, F. Formalizing 100 theorems. http://www.cs.ru.nl/ freek/100/index.html, January 2012. 